\title{
Perturbation expansions of multilocus fixation probabilities for frequency-dependent selection with applications to the Hill-Robertson effect and to the joint evolution of helping and punishment
}

\author{
Laurent Lehmann ${ }^{\mathrm{a}, *}$, François Rousset ${ }^{\mathrm{b}}$ \\ ${ }^{a}$ Department of Biological Sciences, Stanford University, USA \\ ${ }^{\mathrm{b}}$ University of Montpellier II, CNRS, Institut des Sciences de l'Evolution, France
}

\section{A R T I C L E I N F O}

Article history:

Received 16 December 2008

Available online 2 May 2009

\section{Keywords:}

Multilocus models

Fixation probabilities

Coalescence times

Frequency-dependent selection

Hill-Robertson effect

Coevolution of helping and punishment

\begin{abstract}
A B S T R A C T
Natural populations are of finite size and organisms carry multilocus genotypes. There are, nevertheless, few results on multilocus models when both random genetic drift and natural selection affect the evolutionary dynamics. In this paper we describe a formalism to calculate systematic perturbation expansions of moments of allelic states around neutrality in populations of constant size. This allows us to evaluate multilocus fixation probabilities (long-term limits of the moments) under arbitrary strength of selection and gene action. We show that such fixation probabilities can be expressed in terms of selection coefficients weighted by mean first passages times of ancestral gene lineages within a single ancestor. These passage times extend the coalescence times that weight selection coefficients in onelocus perturbation formulas for fixation probabilities. We then apply these results to investigate the Hill-Robertson effect and the coevolution of helping and punishment. Finally, we discuss limitations and strengths of the perturbation approach. In particular, it provides accurate approximations for fixation probabilities for weak selection regimes only $(N s \leqslant 1)$, but it provides generally good prediction for the direction of selection under frequency-dependent selection.
\end{abstract}

(c) 2009 Elsevier Inc. All rights reserved.

\section{Introduction}

In the absence of mutation, any finite population will ultimately become genetically homogeneous as a result of random genetic drift affecting the evolutionary dynamics. The fate of any allele is then either its fixation or loss from the population. For this reason, the fixation probability of a mutant allele is a standard measure used to evaluate its evolutionary success (Crow and Kimura, 1970; Hartl and Clark, 2007; Ewens, 2004; Gillespie, 2004). The fixation probability captures the effects of both genetic drift and natural selection on gene frequency change, from the appearance to the eventual fixation or loss of an allele from the population. But the interaction between genetic drift and selection is complicated and, besides some special cases, population biologists have renounced evaluating fixation probabilities exactly. We are thus left with the necessity of approximating under even the simplest biological scenarios.

Under one-locus genetics, the fixation probability of a mutant allele is generally approximated by three different approaches.

\footnotetext{
* Corresponding author.

E-mail addresses: lehmann@stanford.edu (L. Lehmann), rousset@isem.univ-montp2.fr (F. Rousset).
}

First, the population is assumed to be so large that the interactions between mutants is neglected. This leads to the branching process approximation of fixation probabilities (e.g., Haldane, 1927; Bartlett, 1955; Ewens, 2004). Second, the population is assumed to be large and selection weak, which leads to the diffusion approximation of fixation probabilities (e.g., Crow and Kimura, 1970; Gillespie, 2004; Ewens, 2004). Finally, regardless of population size, selection is assumed to be weak and exact perturbation results are obtained for fixation probabilities near neutrality. This leads to the evaluation of first-order perturbation of fixation probabilities in the strength of selection, which allows one to compute analytically measures of convergence stability for continuous traits under a large spectrum of demographic scenarios in spatially structured populations (e.g., Rousset, 2004; Lessard and Ladret, 2007a; Lehmann, 2008).

For multilocus genetics, there are few results on fixation probabilities when both selection and drift simultaneously affect the evolutionary dynamics, and most results rely on the branching process approximation (e.g., Barton, 1995; Otto and Barton, 1997). Yet how these fixation probabilities depend on population size appears important for Hill-Robertson effects, the evolution of recombination, or the frequency-dependent selection occurring in social evolution (e.g., Hill and Robertson, 1966; Otto and Barton, 2001; Wild and Taylor, 2004; Grafen, 2007). 
In this paper we extend the first-order one-locus perturbation method to an $n$ th-order multilocus perturbation approach. We describe a formalism to calculate systematic perturbation expansions of moments of allelic states (expectations of sets of genes sampled within or between individuals) under arbitrary gene action, which covers epistatic interactions within genomes as well as synergistic interactions between them (thus including social behaviors). This allows us to evaluate $n$ th-order Taylor polynomials in the strength of selection of multilocus fixation probabilities (long-term limits of the moments). We then extend algorithms for deterministic models in order to obtain analytical approximations of fixation probabilities under a Wright-Fisher model of reproduction. We illustrate the method and the approximations by evaluating fixation probabilities of alleles and gametes under two two-locus two-allele settings, one involving the Hill-Robertson effect and the other the coevolution of helping and punishment.

\section{Model}

\subsection{Notation for L-locus fixation probabilities}

The analysis presented in this paper is restricted to a haploid population of constant size $N$ with no further division into demographic classes (e.g. no age structure, separate sexes, or geographic structure). We assume that each individual bears $L$ loci and that a mutant and a resident allele segregate at each locus (i.e., L-locus two-allele model, Slatkin, 1971; Christiansen, 1999), which gives $2^{L}$ different gamete types in the population.

Our notation (Table 1) draws on the general notation for multilocus models in infinite populations of Kirkpatrick et al. (2002) and Roze and Rousset (2008). We denote by $p_{l(i)}$ the frequency ( 0 or 1$)$ of the mutant allele at locus $l$ in individual $i$ of the population. Products of such random variables are noted $p_{V(i)}=\prod_{l \in V} p_{l(i)}$, where $V$ is a set of distinct loci sampled from the same individual in the population (either the $L$ distinct loci or a subset of it). The average of $p_{V(i)}$ over all individuals in the population is denoted

$p_{V}=\frac{1}{N} \sum_{i} p_{V(i)}$,

which is itself a random variable. In the abovementioned works, indicator variables are defined as $\zeta_{l(i)}=p_{l(i)}-\wp_{l(i)}$ relative to some reference values $\wp_{l(i)}$ usually (but not necessarily) taken as the average allele frequency at locus $l$ in the population. This choice of reference values reduces the number of variables that need to be considered in infinite population models, but as no such simplification appears in the finite population model we do not use such variables here.

Sets of loci can also be sampled from distinct individuals; for instance from two individuals, in which case the average of the product of mutant alleles $p_{V(i)} p_{W(j)}$ sampled from the same or distinct loci in two distinct individuals is denoted

$p_{V / W}=\frac{1}{N(N-1)} \sum_{i} \sum_{i \neq j} p_{V(i)} p_{W(j)}$,

where the "/" symbol separates sets of loci sampled from distinct individuals. We will refer to sets of distinct loci sampled from the same individual and sets of similar or distinct loci sampled from different individuals as sets of position. A position refers to a particular locus in a particular context (Kirkpatrick et al., 2002, p. 1729); for instance, the place in the genome where the maternally inherited copy of a given gene resides, or the place in the genome of a neighbor of a given individual where a particular gene affecting a social behavior resides.

More generally sets of positions can be sampled from an arbitrary number of distinct individuals in the population. We thus introduce the random variable $p_{S}$, where $S \equiv S_{1} / S_{2} / \ldots / S_{|S|}$, and where each $S_{i}$ is a set of loci sampled from the same individual
Table 1

List of symbols.

\begin{tabular}{|c|c|}
\hline Symbol & Definition \\
\hline$L$ & Number of loci. \\
\hline$N$ & Population size. \\
\hline$r$ & Recombination rate. \\
\hline$w_{i}$ & Fitness of individual $i$. \\
\hline$f_{i}$ & Fertility of individual $i$. \\
\hline$p_{l(i)}$ & Frequency ( 0 or 1 ) of the mutant allele at locus $l$ in individual $i$. \\
\hline$p_{V(i)}$ & $\begin{array}{l}\text { Frequency ( } 0 \text { or } 1 \text { ) that all loci in the set } V \text { in individual } i \text { carry the } \\
\text { mutant allele. }\end{array}$ \\
\hline$p_{V / W / \ldots}$ & $\begin{array}{l}\text { Average of } p_{V(i)} p_{W(j)} \ldots \text { variables over all individuals in the } \\
\text { population sampled without replacement. }\end{array}$ \\
\hline$p_{v} \widehat{/ w} \widehat{T} \ldots$ & $\begin{array}{l}\text { Average of } p_{V(i)} p_{W(j)} \ldots \text { variables over all individuals in the } \\
\text { population sampled with replacement. }\end{array}$ \\
\hline $\mathrm{E} p_{S}$ & Expectation of $p_{S}$ \\
\hline $\mathbf{p}$ & Vector of the $p_{S}$ variables (vector of random variables). \\
\hline Ep & $\begin{array}{l}\text { Vector of the expectations of the } p_{V} \text { variables (vector of } E p_{S} \\
\text { variables). }\end{array}$ \\
\hline$\wp$ & Vector of expectations of reference variables. \\
\hline $\mathbf{v}$ & Vector of deviations $(\mathbf{v}=$ Ep $-\boldsymbol{\wp})$ \\
\hline d & Vector of the sum of deviations $\left(\mathbf{d} \equiv \sum_{t=0}^{\infty} \mathbf{v}(t)\right)$ \\
\hline $\mathbf{a}_{U}$ & Row of $\boldsymbol{A}$ giving the coefficients describing the dynamics of $p_{U}$. \\
\hline $\mathbf{u}$ & Left eigenvector of $\mathbf{A}^{\circ}$ associated to a unit eigenvalue. \\
\hline e & Right eigenvector of $\mathbf{A}$ associated to a unit eigenvalue. \\
\hline A & Transition matrix of the $\mathrm{E} p_{S}$ variables. \\
\hline $\mathbf{A}_{\wp}$ & Matrix obtained by row replacement of the rows of $\mathbf{A}$. \\
\hline $\mathbf{A}_{\mathrm{c}}$ & $\begin{array}{l}\text { Matrix with each row consisting of the left eigenvector of } \mathbf{A}^{\circ} \\
\text { associated to its unit eigenvalue. }\end{array}$ \\
\hline $\mathbf{Z}$ & Fundamental matrix associated to $\mathbf{A}^{\circ}$. \\
\hline
\end{tabular}

and $|S|$ is the total number of different individuals from which sets of loci have been sampled. The variable $p_{S}$ is obtained in a direct generalization of Eq. (2), by averaging (without replacement) over all $|S|$ distinct individuals. Conditional on some initial state of the population, the expectation of $p_{S}(t)$ at time $t$ is given by

$\mathrm{E}\left[p_{S}(t)\right]=\sum_{\mathbf{p}(t)} p_{S}(t) \operatorname{Pr}(\mathbf{p}(t))$

where $\mathbf{p}(t)$ is a vector collecting the frequencies of the $2^{L}$ gamete types in the population at time $t ; \operatorname{Pr}(\mathbf{p}(t))$ is the distribution of $\mathbf{p}(t)$ at time $t$ conditional on the initial state of the population being $\mathbf{p}(0)$; and the sum is over all possible values of $\mathbf{p}(t)$. We will use the expectation operator notation without brackets as a shorthand for expectation conditional on the initial state $\mathbf{p}(0)$ of the population, e.g., $\mathrm{E}_{S}(t) \equiv \mathrm{E}\left[p_{S}(t)\right]$.

For a single locus denoted $A, E p_{A}$ represents the probability that an individual sampled at random from the population carries the mutant allele at that locus, and $\mathrm{E} p_{\mathrm{A} / \mathrm{A}}$ is the probability that two distinct individuals sampled at random carry the mutant allele. For two loci, denoted $\mathrm{A}$ and $\mathrm{B}, \mathrm{E} \mathrm{p}_{\mathrm{AB}}$ is the probability that a single individual carries the mutant alleles at both loci while $\mathrm{E} p_{\mathrm{AB} / \mathrm{A} / \mathrm{B}}$ is the probability that, among three randomly sampled individuals, one carries the mutant alleles at both loci, another individual carries the mutant allele at locus $A$ and the third individual carries the mutant allele at locus $B$. Note that one can also interpret the subscripts in the $\mathrm{E} p_{S}$ variables as being "alleles" or "gametes" (e.g., $\mathrm{E} p_{\mathrm{A}}$ and $\mathrm{E} p_{\mathrm{AB}}$, are respectively, the frequency of allele $\mathrm{A}$ and gamete $A B$ if the mutant alleles are denoted by the same letter as the locus at which they segregate). The $E p_{S}$ variables can thus be interpreted as moments of allelic states in exactly the same way as is usually done in population genetics (Wright, 1931; Crow and Kimura, 1970; Gillespie, 2004), and we will refer to them as such.

We will generically denote by $p_{U}(t)$ the frequency of a focal gametic combination $U$ at time $t$ in the population, where $U$ is the set of mutant alleles at $L$ distinct loci that can be sampled from the same individual. In the absence of mutation, the focal gametic combination goes either to fixation $\left(p_{U}(\infty)=1\right)$ or is lost from the population $\left(p_{U}(\infty)=0\right)$. The fixation probability $\pi_{U}$ of this gamete is thus its expected frequency in the long-term: 
$\pi_{U}=\mathrm{E} p_{U}(\infty)$. Our main aim in this paper is to obtain the $n$ thorder Taylor polynomials of $\pi_{U}$ with respect to an overall measure $\delta$ of the strength of selection, which may conveniently be defined as the largest (in absolute value) of a set of selection coefficients. The $n$ th-order Taylor polynomial of the fixation probability $\pi_{U}$ of the focal gamete (or of any other quantity $z$ ) will be written as

$\pi_{U}^{[n]}=\sum_{k=0}^{n} \frac{\delta^{k}}{k !} \pi_{U}^{(k)}$,

where $\pi_{U}^{(k)}$ is the $k$ th derivative of $\pi_{U}$ with respect to $\delta$ evaluated at $\delta=0$ ( $n$ th-order perturbation). Generically the superscripts $[n]$ and $(n)$ thus denote, respectively, the $n$ th-order Taylor polynomial and derivative of any quantity. But a special role in our formalization will be played by zero-order Taylor polynomials, which refer to quantities evaluated in the neutral process (no selection, $\delta=0$ ). For ease of presentation, we will use the superscript $\circ$ to identify any quantity evaluated under neutrality (e.g., $\pi_{U}^{[0]}=\pi_{U}^{(0)}=\pi_{U}^{\circ}$ ). Further, in all explicit examples presented below, we will use only first- and second-order derivatives and Taylor polynomials. In order to simplify the presentation of these examples, we will use dot and double dot accents $(\dot{z}, \ddot{z})$ to denote first and second derivatives of any quantity $z$ with respect to $\delta$, and, dot and double dot exponents to denote $\left(z^{*}, z^{*}\right)$, first-, and secondorder Taylor polynomials (e.g., $z^{*}=z^{\circ}+\delta \dot{z}, z^{*}=z^{\circ}+\delta \dot{z}+\delta^{2} \ddot{z} / 2$ ).

We will evaluate $\pi_{U}^{[n]}$ by evaluating the perturbations $\pi_{U}^{(n)}$, which will be carried out by decomposing $\pi_{U}$ into a sum of expected gamete frequency changes over one generation (Rousset, 2003; Lessard and Ladret, 2007b), and then compute the perturbations of expected gamete frequency change. To this end, we denote the change in expected gamete frequency from generation $t$ to $t+1$ by $\Delta \mathrm{E} p_{U}(t) \equiv \mathrm{E} p_{U}(t+1)-\mathrm{E} p_{U}(t)$. With this, the fixation probability can be written as

$$
\begin{aligned}
\pi_{U} & =E p_{U}(\infty) \\
& =p_{U}(0)+\sum_{t=0}^{\infty} \Delta E p_{U}(t) .
\end{aligned}
$$

The change in expected gamete frequency can itself be expanded in terms of conditional changes as

$$
\Delta \mathrm{Ep}_{U}(t)=\sum_{\mathbf{p}(t)} \operatorname{Pr}(\mathbf{p}(t)) \mathrm{E}\left[\Delta p_{U} \mid \mathbf{p}(t)\right],
$$

where $\mathrm{E}\left[\Delta p_{U} \mid \mathbf{p}(t)\right]$ is a shorthand for the expected conditional change in allele frequency $\left[\mathrm{E}\left[p_{U}(t+1) \mid \mathbf{p}(t)\right]-p_{U}(t)\right]$. Our goal is to express $\mathrm{E}\left[\Delta p_{U} \mid \mathbf{p}(t)\right]$ as a linear function of allelic states, and then integrate such expressions over all sample paths. For ease of illustration, we first present the calculations under neutrality and first-order effects of selection, then illustrate these calculations with simple examples, and finally generalize to higher-order perturbations.

\subsection{Neutrality}

Under neutrality, Eq. (6) becomes

$\Delta \mathrm{Ep}_{U}^{\circ}(t)=\sum_{\mathbf{p}(t)} \operatorname{Pr}^{\circ}(\mathbf{p}(t)) \mathrm{E}^{\circ}\left[\Delta p_{U} \mid \mathbf{p}(t)\right]$,

where both the expected change in gamete frequency and the distribution of gamete frequencies are evaluated in the neutral process $(\delta=0)$. As in infinite populations, $\mathrm{E}^{\circ}\left[\Delta p_{U} \mid \mathbf{p}(t)\right]$ can be expressed as $\sum_{X \in X} a_{X}^{\circ} p_{X}(t)$ for some set of positions $\mathcal{X}$, where the $a_{X}^{\circ}$ 's are transmission coefficients (Kirkpatrick et al., 2002) describing the effect of recombination, drift, or both (no selection) on moments of gamete frequency change, and the set $X$ may involve sets of gene copies sampled from the same and from different individuals.

In order to illustrate these notions, we now introduce a twolocus model with a focal gamete consisting of the mutant alleles at loci $A$ and B. Because recombination entails that alleles in a gamete may have distinct parents of origin, we also have to consider the dynamics of $E p_{\mathrm{A} / \mathrm{B}}^{\circ}$ in order to account for that of $\mathrm{E} p_{\mathrm{AB}}^{\circ}$ (Kimura, 1963; Ewens, 2004); where $\mathrm{E} p_{\mathrm{A} / \mathrm{B}}^{\circ}$ is the probability that, among two different individuals, one carries the mutant allele at locus $\mathrm{A}$ and the other carries the mutant allele at locus B. In order to calculate these variables explicitly, we assume a Wright-Fisher random union of gamete model (Karlin, 1968; Ewens, 2004, p. 130). Namely, $N$ haploid parents produce a large number of gametes that fuse randomly to form diploid zygotes (self-fertilization thus occurs with probability $1 / N$ ), which is immediately followed by meiosis with recombination rate $r$ to produce haploid juveniles. Finally, $N$ adults are randomly sampled among the juveniles to form the next generation.

Under this life-cycle, the probability that two genes, sampled from two distinct loci from the same individual, descend from the same individual in the previous generation is $(1-r)+r / N$, where the second term accounts for self-fertilization. In addition, we need the probability that two genes sampled from distinct loci from two distinct individuals, descend from the same individual in the previous generation, which is given by $1 / N$, whereby

$$
\begin{aligned}
\mathrm{E}^{\circ}\left[p_{\mathrm{AB}}(t+1) \mid \mathbf{p}(t)\right]= & \left(1-r\left(\frac{N-1}{N}\right)\right) p_{\mathrm{AB}}(t) \\
& +r\left(\frac{N-1}{N}\right) p_{\mathrm{A} / \mathrm{B}}(t) \\
\mathrm{E}^{\circ}\left[p_{\mathrm{A} / \mathrm{B}}(t+1) \mid \mathbf{p}(t)\right]= & \frac{1}{N} p_{\mathrm{AB}}(t)+\left(\frac{N-1}{N}\right) p_{\mathrm{A} / \mathrm{B}}(t) .
\end{aligned}
$$

By subtracting $p_{\mathrm{AB}}(t)$ on both sides of the first equation and $p_{\mathrm{A} / \mathrm{B}}(t)$ from the second, we obtain

$\mathrm{E}^{\circ}\left[\Delta p_{\mathrm{AB}} \mid \mathbf{p}(t)\right]=-r\left(\frac{N-1}{N}\right)\left(p_{\mathrm{AB}}(t)-p_{\mathrm{A} / \mathrm{B}}(t)\right)$

$\mathrm{E}^{\circ}\left[\Delta p_{\mathrm{A} / \mathrm{B}} \mid \mathbf{p}(t)\right]=\frac{1}{N}\left(p_{\mathrm{AB}}(t)-p_{\mathrm{A} / \mathrm{B}}(t)\right)$,

which shows that the transmission coefficients determining $\mathrm{E}^{\circ}\left[\Delta p_{\mathrm{AB}} \mid \mathbf{p}(t)\right]$ are $-r(N-1) / N$ and $r(N-1) / N$ and those determining $\mathrm{E}^{\circ}\left[\Delta p_{\mathrm{A} / \mathrm{B}} \mid \mathbf{p}(t)\right]$ are $1 / N$ and $-1 / N$.

More generally, we have

$$
\begin{aligned}
\Delta \mathrm{E}_{U}^{\circ}(t) & =\sum_{\mathbf{p}(t)} \operatorname{Pr}^{\circ}(\mathbf{p}(t)) \sum_{X \in X} a_{X}^{\circ} p_{X}(t) \\
& =\sum_{X \in X} a_{X}^{\circ} \sum_{\mathbf{p}(t)} \operatorname{Pr}^{\circ}(\mathbf{p}(t)) p_{X}(t),
\end{aligned}
$$

where the last sum is the expectation of $p_{X}(t)$ in the neutral process, denoted $\mathrm{Ep}_{X}^{\circ}(t)$, so that

$\Delta \mathrm{Ep}_{U}^{\circ}(t)=\sum_{X \in \mathcal{X}} a_{X}^{\circ} \mathrm{Ep}_{X}^{\circ}(t)$,

and when $U$ stands for a single gene copy, all $a_{X}^{\circ}$ are null, whenever there are no mutations.

For the random union of gametes model described by Eq. (9), application of Eq. (11) gives

$\Delta \mathrm{E} p_{\mathrm{AB}}^{\circ}(t)=-r\left(\frac{N-1}{N}\right)\left(\mathrm{E}_{\mathrm{AB}}^{\circ}(t)-\mathrm{Ep}_{\mathrm{A} / \mathrm{B}}^{\circ}(t)\right)$

$\Delta \mathrm{Ep}_{\mathrm{A} / \mathrm{B}}^{\circ}(t)=\frac{1}{N}\left(\mathrm{Ep}_{\mathrm{AB}}^{\circ}(t)-\mathrm{E}_{\mathrm{A} / \mathrm{B}}^{\circ}(t)\right)$. 
By subtracting the second equation from the first one, the measure of expected linkage-disequilibrium (LD) $D_{\mathrm{AB}}^{\circ}(t) \equiv E p_{\mathrm{AB}}^{\circ}(t)-$ $\mathrm{E} p_{\mathrm{A} / \mathrm{B}}^{\circ}(t)$ can be found to satisfy the relation $\Delta D_{\mathrm{AB}}^{\circ}(t)=$ $-(r+(1-r) / N) D_{\mathrm{AB}}^{\circ}(t)$, which yields $D_{\mathrm{AB}}^{\circ}(t)=[(1-r)(N-$ 1) $/ N]^{t} D_{\mathrm{AB}}(0)$, where $D_{\mathrm{AB}}(0)=p_{\mathrm{AB}}(0)-p_{\mathrm{A} / \mathrm{B}}(0)$. By substituting this result into Eq. (12), itself inserted into Eq. (5), we find that the fixation probability of the focal gamete is

$$
\begin{aligned}
\pi_{\mathrm{AB}}^{\circ} & =p_{\mathrm{AB}}(0)-\sum_{t=0}^{\infty} r\left(\frac{N-1}{N}\right) D_{\mathrm{AB}}^{\circ}(t) \\
& =p_{\mathrm{AB}}(0)-\frac{r(N-1) D_{\mathrm{AB}}(0)}{1+r(N-1)} .
\end{aligned}
$$

This result has been derived previously with different approaches [e.g., Karlin and McGregor, 1968, Table 1; Ewens, 2004, eq. 3.138; we mention that the measure of LD defined in this earlier work was $p_{\mathrm{AB}}-p_{\mathrm{A}} p_{\mathrm{B}}=\left(p_{\mathrm{AB}}-p_{\mathrm{A} / \mathrm{B}}\right)(N-1) / N$ since $p_{\mathrm{A}} p_{\mathrm{B}}=p_{\mathrm{AB}} / N+p_{\mathrm{A} / \mathrm{B}}(N-$ 1) $/ N]$.

\subsection{First-order effects}

Taking the derivative on both sides of Eq. (6) with respect to $\delta$ produces the first-order perturbation

$\Delta \dot{\mathrm{E}} p_{U}(t)=\sum_{\mathbf{p}(t)}\left[\dot{\operatorname{Pr}}(\mathbf{p}(t)) \mathrm{E}^{\circ}\left[\Delta p_{U} \mid \mathbf{p}(t)\right]+\operatorname{Pr}^{\circ}(\mathbf{p}(t)) \dot{\mathrm{E}}\left[\Delta p_{U} \mid \mathbf{p}(t)\right]\right]$.

Using the expression $\mathrm{E}^{\circ}\left[\Delta p_{U} \mid \mathbf{p}(t)\right]=\sum_{X \in X} a_{X}^{\circ} p_{X}(t)$ allows us to simplify the first term in Eq. (14) as

$$
\begin{aligned}
\sum_{\mathbf{p}(t)} \dot{\operatorname{Pr}}(\mathbf{p}(t)) \mathrm{E}^{\circ}\left[\Delta p_{U} \mid \mathbf{p}(t)\right] & =\sum_{X \in X} a_{X}^{\circ} \sum_{\mathbf{p}(t)} \dot{\operatorname{Pr}}(\mathbf{p}(t)) p_{X}(t) \\
& =\sum_{X \in X} a_{X}^{\circ} \dot{\mathrm{E}} p_{X}(t)
\end{aligned}
$$

Likewise to the neutral case, $\dot{\mathrm{E}}\left[\Delta p_{U} \mid \mathbf{p}(t)\right]$ can be written as $\sum_{Y \in \mathcal{Y}} \dot{a}_{Y} p_{Y}(t)$ for some set $\mathcal{y}$, where the $\dot{a}_{Y}$ 's are first-order selection coefficients that do not depend on any random variable. For instance, for a one-locus selection model in a random mating population with a mutant allele at locus $A$ having a selective advantage $\delta s_{\mathrm{A}}$ over the resident, we have to first-order

$\dot{\mathrm{E}}\left[\Delta p_{\mathrm{A}}(t) \mid \mathbf{p}(t)\right]=s_{\mathrm{A}}\left(p_{\mathrm{A}}(t)-p_{\mathrm{A}}^{2}(t)\right)$.

The right-hand side of this equation is the classical first-order approximation of the haploid one-locus selection model [Wright, 1969, p. 30; Crow and Kimura, 1970; Gillespie, 2004, i.e., firstorder Taylor series of $\delta s_{A} p_{A}(t)\left(1-p_{A}(t)\right) /\left(1+\delta s_{A} p_{A}(t)\right)$ evaluated at $\delta=0]$. The first-order selection coefficients determining $\dot{\mathrm{E}}\left[\Delta p_{\mathrm{A}}(t) \mid \mathbf{p}(t)\right]$ are thus given by $s_{\mathrm{A}}$ and $-s_{\mathrm{A}}$.

More generally, we have

$$
\begin{aligned}
\sum_{\mathbf{p}(t)} \operatorname{Pr}^{\circ}(\mathbf{p}(t)) \dot{\mathrm{E}}\left[\Delta p_{U} \mid \mathbf{p}(t)\right] & =\sum_{Y \in \mathcal{Y}} \dot{a}_{Y} \sum_{\mathbf{p}(t)} \operatorname{Pr}^{\circ}(\mathbf{p}(t)) p_{Y}(t) \\
& =\sum_{Y \in \mathcal{y}} \dot{a}_{Y} \operatorname{Ep}_{Y}^{\circ}(t),
\end{aligned}
$$

and collecting all terms gives

$\Delta \dot{\mathrm{E}}_{U}(t)=\sum_{Y \in \mathcal{Y}} \dot{a}_{Y} \mathrm{E} p_{Y}^{\circ}(t)+\sum_{X \in X} a_{X}^{\circ} \dot{\mathrm{E}} p_{X}(t)$.

This equation extends the formula for the first-order perturbation of moments of allelic states in a one-locus model (Rousset, 2003; Lessard and Ladret, 2007b) to the multilocus case. For a one-locus model the second term in Eq. (18) is null in the absence of mutation, since all $a_{X}^{\circ}$ are null in that case.

For the one-locus selection model described by Eq. (16), application of Eq. (18) gives

$\Delta \dot{\mathrm{E}} p_{\mathrm{A}}(t)=s_{\mathrm{A}}\left(\mathrm{Ep}_{\mathrm{A}}^{\circ}(t)-\mathrm{Ep}_{\mathrm{A} / \mathrm{A}}^{\circ}(t)\right)$, where $\mathrm{Ep}_{\mathrm{A} / \mathrm{A}}^{\circ}(t)$ is the probability that two individuals sampled with replacement carry the mutant allele $\left(\mathrm{Ep}_{\mathrm{A} / \mathrm{A}}^{\circ}(t)=\mathrm{E}^{\circ}\left[p_{\mathrm{A}}(t)^{2}\right]\right)$. This probability can be expanded as

$\mathrm{Ep}_{\mathrm{A} / \mathrm{A}}^{\circ}(t)=\frac{1}{N} \mathrm{Ep}_{\mathrm{A}}^{\circ}(t)+\left(\frac{N-1}{N}\right) \mathrm{Ep}_{\mathrm{A} / \mathrm{A}}^{\circ}(t)$,

where $E p_{\mathrm{A} / \mathrm{A}}^{\circ}$ is the probability that two individuals sampled without replacement carry the mutant, which gives

$\Delta \dot{\mathrm{Ep}}_{\mathrm{A}}(t)=s_{\mathrm{A}}\left(\frac{N-1}{N}\right)\left(\mathrm{Ep}_{\mathrm{A}}^{\circ}(t)-\mathrm{Ep}_{\mathrm{A} / \mathrm{A}}^{\circ}(t)\right)$.

For the Wright-Fisher scheme of reproduction, the variable $D_{\mathrm{A}}^{\circ}(t) \equiv E p_{\mathrm{A}}^{\circ}(t)-\mathrm{E} p_{\mathrm{A} / \mathrm{A}}^{\circ}(t)$ satisfies $D_{\mathrm{A}}^{\circ}(t)=[(N-1) / N]^{t} D_{\mathrm{A}}(0)$ because $\mathrm{Ep}_{\mathrm{A} / \mathrm{A}}^{\circ}(t+1)=(1 / N) \mathrm{Ep}_{\mathrm{A}}^{\circ}(t)+[(N-1) / N] \mathrm{E} p_{\mathrm{A} / \mathrm{A}}^{\circ}(t)$, where $1 / N$ is the probability that two individuals descend from the same parent. By substituting this result into Eq. (21), itself inserted into Eq. (5), we find that the first-order Taylor polynomial of the fixation probability is given by

$$
\begin{aligned}
\pi_{\dot{\mathrm{A}}} & =p_{\mathrm{A}}(0)+\delta\left[\sum_{t=0}^{\infty} s_{\mathrm{A}}\left(\frac{N-1}{N}\right) D_{\mathrm{A}}^{\circ}(t)\right] \\
& =p_{\mathrm{A}}(0)+\delta s_{\mathrm{A}}\left(\frac{N-1}{N}\right) N D_{\mathrm{A}}(0) .
\end{aligned}
$$

This result has been derived previously, and when there is initially a single mutant allele in the population we have $p_{\mathrm{A}}(0)=$ $D_{\mathrm{A}}(0)=1 / N$ (Hill, 1972, eq. 7; Rousset and Billiard, 2000; Lessard and Ladret, 2007b). As pointed out by Lessard and Ladret, we can compute the derivative of the ultimate expected frequency (fixation probability) as the sum of expected derivatives in each generation if the series of expected derivatives converges uniformly in a neighborhood of $\delta=0$. They showed this for the one-locus Cannings exchangeable model, and this follows more generally from Eq. (A.10) (see explanations in the Appendix right after this equation).

\section{4. nth-order effects}

\subsubsection{Recursions for moments of allelic states}

The $n$ th-order perturbation of the conditional expected change in gamete frequency can be written as

$$
\mathrm{E}^{(n)}\left[\Delta p_{U} \mid \mathbf{p}(t)\right]=\sum_{X_{n} \in X_{n}} a_{X_{n}}^{(n)} p_{X_{n}}(t)
$$

for some set $\mathcal{X}_{n}$, where the $a_{X_{n}}^{(n)}$ are $n$ th-order selection coefficients that do not depend on any random variable. For any $n \geqslant 0$, these coefficients satisfy

$$
\sum_{X_{n} \in X_{n}} a_{X_{n}}^{(n)}=0
$$

because, when every individual in the population bears the same genotype (e.g., all $p_{X_{n}}=1$ ), no systematic change in gamete frequency can occur over a selection, recombination or migration phase. This logic applies to the expected change in frequency of any genotype for any L-locus model, and for any submodel involving only a subset of loci of positions. Thus, in a three-locus model, $\sum_{W_{n} \in W_{\eta}} a_{W_{n}}^{(n)}=0$ for every subset $w_{n}$ of all sets of positions involving only one locus, or at most two loci. This implies that $\sum_{W \in W_{n}} a_{W_{n}}^{(n)}=0$ for every subset $w_{n}$ of sets of positions each involving exactly two given loci. Thus with 3 loci, 7 nonoverlapping sets of coefficients each add up to zero, one set for each locus, one set for each pair of locus, and the set of coefficients involving all three loci. 
Differentiating the left- and right-hand side of Eq. (6) $n$ times with respect to $\delta$, using Leibniz's rule for the differentiation of products, inserting Eq. (23), and rearranging allows us to write

$E p_{U}^{(n)}(t+1)=E p_{U}^{(n)}(t)+\sum_{j=0}^{n}\left(\begin{array}{l}n \\ j\end{array}\right) \sum_{X \in X_{j}} a_{X}^{(j)} E p_{X}^{(n-j)}(t)$.

On substitution of this equation into the expansion $\mathrm{Ep}_{U}^{[n]}(t+1)$ (see Eq. (4)), we obtain after rearrangements a recursion on Taylor polynomials:

$\mathrm{E} p_{U}^{[n]}(t+1)=\mathrm{E} p_{U}^{[n]}(t)+\sum_{j=0}^{n} \frac{\delta^{j}}{j !} \sum_{X \in X_{j}} a_{X}^{(j)} \mathrm{E}_{X}^{[n-j]}(t)$.

Since a similar recursion can be written for the Taylor polynomials $\mathrm{E} p_{X}^{[n-j]}(t)$ of any set of allelic states $X$ appearing on the right-hand side of Eq. (26), the recursion for $\mathrm{E} p_{U}^{[n]}$ can be closed (the same logic also applies to Eq. (25)). More generally, one can evaluate in this way the $n$th Taylor polynomial $E p_{S}^{[n]}$ of any moment of allelic state $S$ in the population, be it the complete genetic description of the population.

\subsubsection{Matrix representation}

The dynamics of the Taylor polynomials $\mathrm{E} p_{U}^{[n]}(t)$ of the focal gamete (or more generally of any moment of allelic state) as described by Eq. (26) are linear in the expectations, which suggests that they can be evaluated using matrix algebra. To this end, we gather all the moments affecting the dynamics of $E p_{U}^{[n]}(t)$ into a vector, except the lower-order polynomials of the highest-order moment of any set $S$ of positions involved in the recursion (if we have $\mathrm{Ep}_{S}(t)$, we do not consider $\mathrm{E}_{S}(t)$ nor $\mathrm{E} p_{S}^{\circ}(t)$ in the vector). We then augment this vector with any additional moment required to close the system of recursions. The vector gathering the total set $\checkmark$ of positions closing the system is denoted $\operatorname{Ep}(t) \equiv\left(\operatorname{Ep}_{S}(t)\right)_{S \in S}$, and it satisfies the recursion

$\operatorname{Ep}(t+1)=\operatorname{AEp}(t)$,

for some matrix A collecting the transmission and selection coefficients appearing in Eq. (26). Importantly, Eq. (27) is not a direct matrix formulation of Eq. (26), as it involves only the highest-order polynomial of any set of positions $S$ involved in Eq. (26). This allows us to shrink the size of $\mathbf{A}$, but with the charge of carrying terms in excess powers in $\delta$, which can subsequently be disposed of by taking a Taylor series (see below).

In order to illustrate these concepts we use the two explicit examples introduced above. For the two-locus neutral random union of gamete model (Eq. (12)), we need the vector $\operatorname{Ep}(t) \equiv$ $\left(\mathrm{Ep}_{\mathrm{AB}}^{\circ}(t), \mathrm{Ep}_{\mathrm{A} / \mathrm{B}}^{\circ}(t)\right)$ to follow the dynamics of $\mathrm{Ep}_{\mathrm{AB}}^{\circ}(t)$. The dynamics of this vector is described by the matrix with neutral coefficients given by

$\mathbf{A}^{\circ}=\left(\begin{array}{cc}1-r\left(\frac{N-1}{N}\right) & r\left(\frac{N-1}{N}\right) \\ \frac{1}{N} & \frac{N-1}{N}\end{array}\right)$.

For the the one-locus selection model (Eq. (21)), we need the vector $\mathrm{Ep}(t) \equiv\left(E p_{\mathrm{A}}(t), \mathrm{E} p_{\mathrm{A} / \mathrm{A}}^{\circ}(t)\right)$ to track the dynamics of $\mathrm{E} p_{\mathrm{A}}(t)$. The dynamics of this vector can be described by the transition matrix

$\mathbf{A}^{\cdot}=\mathbf{A}^{\circ}+\delta \dot{\mathbf{A}}$,

where

$\mathbf{A}^{\circ}=\left(\begin{array}{cc}1 & 0 \\ \frac{1}{N} & \frac{N-1}{N}\end{array}\right)$, gathers all neutral coefficients, while

$\dot{\mathrm{A}}=\left(\begin{array}{cc}s_{\mathrm{A}}\left(\frac{N-1}{N}\right) & -s_{\mathrm{A}}\left(\frac{N-1}{N}\right) \\ 0 & 0\end{array}\right)$

gathers all first-order selection coefficients. More generally, we can express $\mathbf{A}$ as a linear combination of matrices $\mathbf{A}^{(n)}$ gathering all the $n$ th-order $a_{X_{n}}^{(n)}$ selection coefficients.

These examples illustrate that $\mathbf{A}^{\circ}$ is a row stochastic matrix (each element $i j$ is either null or positive, and the rows sum up to one, which entails at least one eigenvalue 1). But more generally, $\mathbf{A}^{\circ}$ may involve several independent neutral systems, so that $\mathbf{A}^{\circ}$ is a diagonal block matrix, where each block is the transition matrix of a Markov chain (Grinstead and Snell, 1997; Iosifescu, 2007), one for each set of sets of positions involving exactly a given number of distinct loci. For instance, for a two-locus model we may need to evaluate $\mathrm{Ep}^{\circ}(t) \equiv\left(\mathrm{Ep}_{\mathrm{A}}^{\circ}(t), \mathrm{Ep}_{\mathrm{A} / \mathrm{A}}^{\circ}(t), \mathrm{Ep}_{\mathrm{AB}}^{\circ}(t), \mathrm{E} p_{\mathrm{A} / \mathrm{B}}^{\circ}(t)\right)$, whose dynamics under Wright-Fisher random union of gametes is described by the two-block diagonal matrix

$$
\mathbf{A}^{\circ}=\left(\begin{array}{cccc}
1 & 0 & 0 & 0 \\
\frac{1}{N} & \frac{N-1}{N} & 0 & 0 \\
0 & 0 & 1-r\left(\frac{N-1}{N}\right) & r\left(\frac{N-1}{N}\right) \\
0 & 0 & \frac{1}{N} & \frac{N-1}{N}
\end{array}\right)
$$

(see also Eq. (A.2) of the Appendix). The element $i j$ of $\mathbf{A}^{\circ}$ gives the probability that a set of genes sampled in the set of positions $i$ descend from the set of positions $j$ in the previous generation. As can be seen from Eq. (32) (or Eq. (A.2)), all elements $i j$ of $\mathbf{A}^{\circ}$ are null when $i$ and $j$ involves sets of positions with a different number of loci (one loci is involved in the set of positions $\{A, A / A\}$ while two distinct loci are involved in the set of positions $\{A B, A / B\}$ ). All properties of $\mathbf{A}^{\circ}$ can thus be deduced from the properties of each block because each neutral system in a block is a Markov chain independent from those in the other blocks, and we assume, without obvious biological restrictions, that each block is a mixing Markov chain (Iosifescu, 2007, p. 126; a Markov chain is mixing if it eventually converges to a stable stationary distribution). These examples also illustrate that in the presence of selection, the $\mathbf{A}$ matrix no longer has the block structure of $\mathbf{A}^{\circ}$, and is no longer a stochastic matrix as some of its elements can be negative (e.g., Eq. (29)), though all its row sums are still equal to 1 , so it still has eigenvalue 1 .

\subsection{Fixation probabilities and mean first passage times}

\subsubsection{Taylor polynomials of fixation probabilities}

The Taylor polynomial $\pi_{U}^{[n]}$ of the fixation probability is given by $\mathrm{E}_{U}^{[n]}(\infty)$; namely, the long-term limit of the moments in the absence of mutation, which can either be directly obtained from the vector $\mathrm{Ep}^{[n]}(\infty)$ (Eq. (27)) or by summing up $\Delta \mathrm{E} p_{U}^{[n]}(t)$ from the time of the appearance of the mutant gamete until its eventual fixation in, or loss from, the population (Eq. (5), see also Eqs. (13) and (22)). While the first approach seems at first glance more direct, it involves the evaluation of the eigenvectors of $\mathbf{A}$ associated with its unit eigenvalues, and the deletion of the excess powers in $\delta$ mentioned above by taking a Taylor series. As will be shown below, the second approach involves only the inversion of a matrix considered at neutrality (derived from $\mathbf{A}^{\circ}$ ). This approach has been used previously in the one-locus model because it allows one to express fixation probabilities in terms of selection coefficients and coalescence times (Rousset, 2003; Lessard and Ladret, 2007b). 
The relationship between coalescence times and measures of genetic association (Slatkin, 1991), such as relatedness coefficients, then gave a link between fixation probabilities and concepts of inclusive fitness theory (Hamilton, 1964, 1970). We will extend this interpretation of fixation probabilities to multilocus models.

By iterating Eq. (27) one could try to express effects of selection in terms of $\sum_{t=0}^{\infty} \mathbf{A}^{t}=(\mathbf{I}-\mathbf{A})^{-1}$ where $\mathbf{I}$ is the identity matrix. However, matrix inversion is not directly applicable to the I - A matrix because $\mathbf{A}$ has unit eigenvalues. Correspondingly, expressions like $\sum_{t} \Delta \dot{\mathrm{E}} p_{U}(t)$ generate sums like $\sum_{t} \sum_{Z \in Z} \dot{a}_{Z} \mathrm{Ep} p_{Z}^{\circ}(t)$ (e.g. Eq. (18)), which cannot be written as $\sum_{Z \in Z} \dot{a}_{Z} \sum_{t} \mathrm{E} p_{Z}^{\circ}(t)$ because each sum over $t$ is infinite. Still, the whole expression $\sum_{t} \sum_{Z \in Z} \dot{a}_{Z} E p_{Z}^{\circ}(t)$ is finite, which is made possible by the fact that $\sum_{Z \in \mathcal{Z}} \dot{a}_{Z}=0$, and which was implicitly used in our two examples (see Eqs. (13) and (22)). We may thus consider a reference value $\wp_{z}^{\circ}$ such that $\sum_{t} \sum_{Z \in \mathcal{Z}} \dot{a}_{Z} \mathrm{E} p_{Z}^{\circ}(t)$ can be written as $\sum_{t} \sum_{Z \in Z} \dot{a}_{Z} v_{Z}^{\circ}(t)$, where $v_{Z}^{\circ}(t) \equiv \mathrm{E}_{Z}^{\circ}(t)-\wp_{Z}^{\circ}(t)$ is a centered variable. If every $\sum_{t} v_{Z}^{\circ}(t)$ is finite (for instance because $v_{Z}^{\circ}(t)$ vanishes geometrically fast) we can evaluate $\sum_{Z \in Z} \dot{a}_{Z} \sum_{t} v_{Z}^{\circ}(t)$. For instance, the variables $D_{\mathrm{AB}}^{\circ}(t)$ and $D_{\mathrm{A}}^{\circ}(t)$ in Eqs. (13) and (22) vanish geometrically fast and allowed us to evaluate the sum. Further, from Eqs. (5), (25) and (26), we can compute the $n$th derivative or the $n$th Taylor polynomial of the fixation probability as, respectively,

$\pi_{U}^{(n)}=\sum_{j=0}^{n}\left(\begin{array}{l}n \\ j\end{array}\right) \mathbf{a}_{U}^{(j)} \cdot \mathbf{d}^{(n-j)}$,

and

$\pi_{U}^{[n]}=p_{U}(0)+\sum_{j=0}^{n} \frac{\delta^{j}}{j !}\left(\mathbf{a}_{U}^{(j)} \cdot \mathbf{d}^{[n-j]}\right)$,

where - denotes the dot product, $\mathbf{a}_{U}$ is the row of $\mathbf{A}$ gathering the coefficients describing the dynamics of $\mathrm{Ep}_{U}$ and

$\mathbf{d} \equiv \sum_{t=0}^{\infty} \mathbf{v}(t)$

is the sum of the vectors $\mathbf{v}(t) \equiv\left(v_{S}(t)\right)_{S \in S} \equiv\left(E p_{S}(t)-\wp_{S}(t)\right)_{S \in S}$ (now considered out of neutrality).

In the Appendix ("Recursion for $\mathbf{v}(t)$ "), we show that appropriate choices for the reference $\wp_{\mathcal{Z}}^{\circ}$ values are the values $\operatorname{Ep}_{U}(t)$ for the reference set $R$ in which all loci involved in $S$ are sampled in the same individual (e.g. if $S=A / A B / C$ then three loci are involved and $R=\mathrm{ABC}$; for the two-locus random union of gamete model presented above $R=\mathrm{AB}$, while for the one-locus model $R=\mathrm{A}$ ). Then, $\mathbf{v}(t)$ satisfies the recursion $\mathbf{v}(t+1)=\left(\mathbf{A}-\mathbf{A}_{\wp}\right) \mathbf{v}(t)$, where $\mathbf{A}_{\wp}$ is a matrix obtained directly from the elements of $\mathbf{A}$ (no new element needs to be evaluated separately), and such that $\mathbf{I}-\mathbf{A}+\mathbf{A}_{\wp}$ is non-singular, whereby

$\mathbf{d}=\left(\mathbf{I}-\mathbf{A}+\mathbf{A}_{\wp}\right)^{-1} \mathbf{v}(0)$.

The Taylor polynomials of $\mathbf{d}$ appearing in Eq. (34) consist of the perturbations $\mathbf{d}^{(n)}$ of $\mathbf{d}$, which involve $n$ th-order derivatives of the matrix $\left(\mathbf{I}-\mathbf{A}+\mathbf{A}_{\wp}\right)^{-1}$, which can themselves be expressed as a linear combination of the matrices $\mathbf{A}^{(n)}$ gathering the selection coefficients weighted by powers of $\left(\mathbf{I}-\mathbf{A}^{\circ}+\mathbf{A}_{\wp}^{\circ}\right)^{-1}$ (e.g. Eq. (A.14); Singh, 1990). Hence, only inversions of matrices computed under neutrality $(\delta=0)$ are required to obtain the perturbations expansions of the fixation probabilities.

\subsubsection{Forward and backward neutral processes}

In the one-locus model, the elements of $\mathbf{d}^{\circ}$ are mean coalescence times between gene copies sampled at two or more different homologous positions. We will now extend this relationship and show that it holds more generally, for any set of positions in a multilocus setting. For ease of presentation, consider that $\mathbf{A}^{\circ}$ consists of a single neutral system in the following (as all the expressions hold with all matrices being diagonal block matrices where each block satisfies the given arguments). It is first useful to recall that the transition matrix $\mathbf{A}^{\circ}$ used in $\mathrm{Ep}^{\circ}(t+$ 1) $=\mathbf{A}^{\circ} \mathbf{E p}^{\circ}(t)$ describes a forward process; that is, the change in moments of allelic states from the present, $t=0$, forwards in time. But $\mathbf{A}^{\circ}$ can also be used to describe a backward process

$\mathbf{u}(h+1)=\mathbf{u}(h) \mathbf{A}^{\circ}$,

where $h$ proceeds from the present, $h=0$, backwards in time, and where element $u_{V}(h)$ of $\mathbf{u}(h)$ gives the probability that the ancestral lineages of a set of genes sampled from a given set of positions in the present will be in the set of positions $V$ at time $h$. For instance, for the two-locus random union of gamete model (Eq. (28)), we have $\mathbf{u}(h)=\left(u_{\mathrm{AB}}(h), u_{\mathrm{A} / \mathrm{B}}(h)\right)$, where $u_{\mathrm{AB}}(h)$ is the probability that the ancestral lineages of two genes sampled at loci $A$ and $B$ will reside in those loci sampled from the same individual at time $h$, while $u_{\mathrm{A} / \mathrm{B}}(h)$ is the probability that these lineages will reside in loci sampled from two different individuals. For the onelocus model (Eq. (30)), we have $\mathbf{u}(h)=\left(u_{\mathrm{A}}(h), u_{\mathrm{A} / \mathrm{A}}(h)\right)$, where $u_{\mathrm{A}}(h)$ is the probability that the ancestral lineages of two genes sampled at loci A will be in the same individual at time $h$, while $u_{\mathrm{A} / \mathrm{A}}(h)$ is the probability that these ancestral lineages will reside in loci sampled from different individuals.

In a one-locus situation, ancestral lines can only coalesce in the same ancestor so that $u_{\mathrm{A}}(\infty)=1$ while $u_{\mathrm{A} / \mathrm{A}}(\infty)=0$ (the Markov chain describing the neutral system is absorbing). But in the presence of recombination, gene lineages residing in the same individual can also reach positions in different individuals so that the circulation of several distinct gene lineages between several gene positions eventually reaches a stationary distribution. In general there are thus both transient states (all those involving more than one position at a locus) and a recurrent set of states. The stationary distribution ( probability vector $\mathbf{u}$ ) over all ancestral sets of lineages is given by the left unit eigenvector of the Markov transition matrix (i.e., $\mathbf{u}=\mathbf{u} \mathbf{A}^{\circ}$ ). This stationary distribution provides the mean recurrence time $\left(T_{S}=1 / u_{S}\right)$; that is, the mean number of steps it takes (looking backwards in time) for the ancestral lines of a set of genes, presently in the set of positions $S$, to return to $S$. But the elements of $\mathbf{A}^{\circ}$ also allow us to evaluate $T_{S V}$, which is the expected number of steps it takes for the ancestral lines of a set of genes in $S$ to reach the set of positions $V$, and where by definition $T_{S S}=0$ (thus being distinct from the recurrence time $T_{S}$ ).

For recurrent states $S$, we show in the Appendix that the elements $S V$ of $\left(\mathbf{I}-\mathbf{A}^{\circ}+\mathbf{A}_{\wp}^{\circ}\right)^{-1}$ are given by $\left(T_{R V}-T_{S V}\right) u_{V}+\delta_{R V}$, where $\delta_{R V}$ is the usual Kronecker's delta notation (Eqs. (A.15)(A.21)). With this result, we find that for any recurrent state $S$, the Sth element of $\mathbf{d}^{\circ}$ is

$d_{S}^{\circ}=\sum_{V \in \mathcal{V}}\left(T_{R V}-T_{S V}\right) u_{V} p_{V}(0)$

where we again consider the reference set $R$ to be the set in which all loci involved in $S$ are sampled in the same individual (moreover, in the same gamete for diploid organisms). This shows that, regardless of the strength of selection, and the complexity of the interaction within and between genomes, $\pi_{U}$ can be expressed in terms of selection coefficients weighted by mean first passage times; that is, in terms of genealogical ties.

For transient states $S$, Eq. (38) is extended in terms of the mean time $T_{S V}$ spent in transient states $V$ before absorption in the recurrent set (mean sojourn time), and of the probability distribution of transient states through which the recurrent set is first entered, all conditional to the initial state $S$ (Eq. (A.27)). Such sojourn times can further be related to coalescence times when 
the reference set $R$ is absorbing, as occurs in the one-locus model. Indeed, the mean coalescence time $T_{S R}$ is the mean time before the chain is absorbed in $R$, given that it starts in $S$, and $T_{S R}$ is thus the sum over all mean times spent in the different transient states before coalescence in state $R$ occurs (i.e., $T_{S R}=\sum_{\operatorname{trans} V \in \mathcal{V}} T_{S V}$, where the sum is over all transient states, Eq. (A.29)). In particular, for the one-locus case in which there is a single initial mutant allele at locus, say $\mathrm{A}$, the reference set is $R=\mathrm{A}$, while $p_{\mathrm{A}}(0)=1 / N$ and $p_{V}(0)=0$ otherwise. With this we have $d_{S}^{\circ}=-T_{S A} / N$ (which can be obtained directly from Eq. (38) for this special case or, more generally, from Eq. (A.28)), and we have

$\dot{\pi}_{\mathrm{A}}=-\frac{1}{N} \sum_{S \in \delta} \dot{a}_{\mathrm{AS}} T_{S \mathrm{~A}}$,

which matches previous results (Rousset, 2003, eq. 15; Lessard and Ladret, 2007b, eqs. 48-50). These results further reduce when selection depends only on the genic variance in the population as described by Eq. (16). Then $s=\{\mathrm{A}, \mathrm{A} / \mathrm{A}\}$, and for a random mating population $T_{\mathrm{AA}}=0, T_{(\mathrm{A} / \mathrm{A}) \mathrm{A}}=N$, and $\dot{\mathbf{a}}_{\mathrm{A}} \equiv\left(\dot{a}_{\mathrm{AA}}, \dot{a}_{(\mathrm{A} / \mathrm{A}) \mathrm{A}}\right)=$ $\left(s_{\mathrm{A}}(N-1) / N,-s_{\mathrm{A}}(N-1) / N\right)$. On substitution of these variables into Eq. (39), we recover the result established above (Eq. (22)):

$\dot{\pi}_{\mathrm{A}}=s_{\mathrm{A}}\left(\frac{N-1}{N}\right)$.

\section{Applications}

We will present applications of our results by evaluating explicitly fixation probabilities of alleles and gametes for twolocus two-alleles models under the Wright-Fisher random union of gamete life-cycle introduced above (Ewens, 2004). For this purpose, we first detail the construction of multilocus recursions for this reproductive scheme.

\subsection{Recursions for Wright-Fisher panmictic population}

In order to construct $\mathbf{A}$ under our life cycle assumptions, we need recursions for $E p_{S}$ variables over the reproduction and the recombination phase. Recall that $S=S_{1} / S_{2} / \cdots / S_{|S|}$, where each $S_{i}$ is a set of loci sampled from the same individual and $|S|$ denotes the total number of different individuals in $S$. The recursions for the $\mathrm{E} p_{S}$ variables must take finite population size into account, and can be directly obtained from recursions derived by Roze and Rousset (2008, eqs. 40-42) for the infinite island model with finite deme size, when the dispersal rate is set to zero.

The $|S|$ different individuals from which sets of positions in $S$ have been sampled in a given offspring generation may have descended from $|X|$ different individuals from the parental generation $(1 \leqslant|X| \leqslant|S|)$. These parents carry the sets of positions $S$ in a different way because coalescence merges and recombination rearranges sets of positions. The reorganized sets of positions in the parental generation is denoted $X=X_{1} / X_{2} / \cdots / X_{|X|}$, and the contribution of parent $i$ to the offspring generation depends on its fitness (expected number of offspring reaching the adult stage), which can be written as $w_{i}=f_{i} / f$, where $f_{i}$ is the relative fecundity of individual $i$ and $f=\frac{1}{N} \sum_{k} f_{k}$ is the average relative fecundity of individuals in the population.

In order to simplify the presentation of the recursions of moments of allelic states, we drop from now on the time indices $t$ attached to any quantity $z$, and use a prime $\left(z^{\prime}\right)$ to denote any quantity after the reproduction stage and a double prime $\left(z^{\prime \prime}\right)$ to denote any quantity after the recombination stage. Hence, a recursion over the life cycle goes from $z$ to $z^{\prime \prime}$, which corresponds to go from $z(t)$ to $z(t+1)$ in the notation used in the previous sections.
With this and the above, the conditional expected moments of allelic state after reproduction is given by

$\mathrm{E}\left[p_{S}^{\prime} \mid \mathbf{p}\right]=\sum_{X \in C_{S}} P(S, X) \mathrm{E}_{i_{1}, i_{2} \neq i_{1}, \ldots}\left[w_{i_{1}}^{\left|y_{1}\right|} w_{i_{2}}^{\left|y_{2}\right|} \cdots p_{X_{1}\left(i_{1}\right)} p_{X_{2}\left(i_{2}\right)} \cdots\right]$

where $\mathrm{E}_{i_{1}, i_{2} \neq i_{1}, \ldots}$ means the average over all parents $i_{1}, i_{2} \neq i_{1}, \ldots$ of the population; $C_{S}$ is the set of all ways of allocating $|S|$ offspring to $|X|$ parents, with $\left|y_{1}\right|$ offspring coming from parent $i_{1},\left|y_{2}\right|$ offspring coming from parent $i_{2}, \ldots$ (i.e. $|S|=\sum_{i=1}^{|X|}\left|y_{i}\right|$ and $C_{S}$ is the set partition of $\{1,2,3, \ldots,|S|\}$ into non-empty subsets); and $P(S, X)$ is the probability of partition $X$ (coalescence event), which is given by

$P(S, X)=\frac{1}{N^{|S|-|X|}} \prod_{y=1}^{|X|-1}\left(1-\frac{y}{N}\right)$.

For instance the expected frequency $p_{U}$ of gamete $U$ after reproduction is

$\mathrm{E}\left[p_{U}^{\prime} \mid \mathbf{p}\right]=\mathrm{E}_{i}\left[w_{i} p_{U(i)}\right]$,

while that of $p_{U / V}$ is

$\mathrm{E}\left[p_{U / V}^{\prime} \mid \mathbf{p}\right]=\frac{1}{N} \mathrm{E}_{i}\left[w_{i}^{2} p_{U V(i)}\right]+\left(\frac{N-1}{N}\right) \mathrm{E}_{i, k \neq i}\left[w_{i} w_{k} p_{U(i)} p_{V(k)}\right]$

Over the recombination phase one has

$\mathrm{E}\left[p_{S}^{\prime \prime} \mid \mathbf{p}^{\prime}\right]=\sum_{U V=S_{1}} \sum_{W X=S_{2}} \cdots \sum_{Y Z=S_{|S|}} r_{U \mid V} r_{W \mid X} \ldots r_{Y \mid Z} p_{U / V / W / X / \ldots / Y / Z}^{\prime}$

where $r_{A \mid B}$ is the probability that, in a meiotic product, the set $A$ of loci comes from one parent while the set $B$ comes from another parent. The sum over $U V=S_{1}$ means the sum over all partitions $U$ and $V$ of the set $S_{1}$ with the partition $\left\{S_{1}, \varnothing\right\}$ included.

As in the structured population case (Roze and Rousset, 2008), further analysis proceeds by expressing the fitness functions $w$ in terms of the genotypes at different loci in different individuals (as described by the $p$ variables, see example below), and expanding such expressions to various orders of approximation with respect to the selection coefficients. Because the calculations are tedious to perform by hand, Roze and Rousset (2008) implemented Eqs. (41) and (45) in Mathematica (Wolfram, 2003), by which the evaluation of the transition matrix $\mathbf{A}$ to leading order in selection coefficients can be automated. We also implemented Eq. (34) in Mathematica in order to obtain directly the fixation probability to a given order of accuracy.

\subsection{Hill-Robertson effect}

We now consider two loci, where the mutant allele at locus A has a selective advantage $\delta s_{A}$ over the resident, and the mutant allele at locus $\mathrm{B}$ locus has a selective advantage $\delta s_{\mathrm{B}}$ over the resident. We assume that alleles affect fecundity in a multiplicative way: $f_{i}=\left(1+\delta s_{\mathrm{A}} p_{\mathrm{A}(i)}\right)\left(1+\delta s_{\mathrm{B}} p_{\mathrm{B}(i)}\right)$, which entails that $f=$ $1+\delta s_{\mathrm{A}} p_{\mathrm{A}}+\delta s_{\mathrm{B}} p_{\mathrm{B}}+\delta^{2} s_{\mathrm{A}} s_{\mathrm{B}} p_{\mathrm{AB}}$. With this, the fitness of individual $i$ is

$w_{i}=\frac{\left(1+\delta s_{\mathrm{A}} p_{\mathrm{A}(i)}\right)\left(1+\delta s_{\mathrm{B}} p_{\mathrm{B}(i)}\right)}{1+\delta s_{\mathrm{A}} p_{\mathrm{A}}+\delta s_{\mathrm{B}} p_{\mathrm{B}}+\delta^{2} s_{\mathrm{A}} s_{\mathrm{B}} p_{\mathrm{AB}}}$

\subsubsection{First-order effects on $\pi_{\mathrm{A}}$}

In order to evaluate $\pi_{\dot{A}}$, we need to compute the recursion for $\mathrm{Ep} \cdot \mathrm{A}$. This is obtained by inserting the first-order Taylor polynomial of Eq. (46),

$w_{i}^{*}=1+\delta s_{\mathrm{A}}\left(p_{\mathrm{A}(i)}-p_{\mathrm{A}}\right)+\delta s_{\mathrm{B}}\left(p_{\mathrm{B}(i)}-p_{\mathrm{B}}\right)$, 
into Eq. (43), whereby

$$
\begin{aligned}
\mathrm{E}^{*}\left[p_{\mathrm{A}}^{\prime} \mid \mathbf{p}\right] & =\mathrm{E}_{i}\left[p_{\mathrm{A}(i)}+\delta s_{\mathrm{A}}\left(p_{\mathrm{A}(i)}^{2}-p_{\mathrm{A}} p_{\mathrm{A}(i)}\right)+\delta s_{\mathrm{B}}\left(p_{\mathrm{B}(i)} p_{\mathrm{A}(i)}-p_{\mathrm{B}} p_{\mathrm{A}(i)}\right)\right] \\
& =p_{\mathrm{A}}+\delta s_{\mathrm{A}}\left(p_{\mathrm{A}}-p_{\mathrm{A}} p_{\mathrm{A}}\right)+\delta s_{\mathrm{B}}\left(p_{\mathrm{AB}}-p_{\mathrm{A}} p_{\mathrm{B}}\right)
\end{aligned}
$$

where the second line is obtained by noting that $p_{\mathrm{A}(i)}^{2}=p_{\mathrm{A}(i)}$ and $p_{\mathrm{B}(i)} p_{\mathrm{A}(i)}=p_{\mathrm{AB}(i)}$. Integrating over all sample paths then produces

$\mathrm{E} p_{\mathrm{A}}^{{ }^{\prime}}=\mathrm{E} p_{\mathrm{A}}^{\cdot}+\delta s_{\mathrm{A}}\left(\mathrm{E} p_{\mathrm{A}}^{\circ}-\mathrm{E}_{\mathrm{A} / \mathrm{A}}^{\circ}\right)+\delta s_{\mathrm{B}}\left(\mathrm{E}_{\mathrm{AB}}^{\circ}-\mathrm{E}_{\mathrm{A} / \mathrm{B}}^{\circ}\right)$,

where

$\mathrm{Ep}_{V / W}^{\circ} \equiv \frac{1}{N} \mathrm{Ep}_{V W}^{\circ}+\left(\frac{N-1}{N}\right) \mathrm{Ep}_{V / W}^{\circ}$,

which gives after simplification

$\mathrm{E} p_{\mathrm{A}}^{{ }^{\prime}}=\mathrm{E} p_{\mathrm{A}}^{\cdot}+\delta\left(\frac{N-1}{N}\right)\left[s_{\mathrm{A}}\left(\mathrm{E}_{\mathrm{A}}^{\circ}-\mathrm{E}_{\mathrm{A} / \mathrm{A}}^{\circ}\right)+s_{\mathrm{B}}\left(\mathrm{E} p_{\mathrm{AB}}^{\circ}-\mathrm{E}_{\mathrm{A} / \mathrm{B}}^{\circ}\right)\right]$

Recombination does not affect the dynamics of $\mathrm{Ep}_{\mathrm{A}}{ }^{\prime}$, thereby

$\mathrm{E} p_{\mathrm{A}}^{\prime \prime}=\mathrm{E} p_{\mathrm{A}}^{\prime}$.

These equations show that we need the additional neutral expectations, $\mathrm{E} p_{\mathrm{AB}}^{\circ}, \mathrm{E} p_{\mathrm{A} / \mathrm{A}}^{\circ}$ and $\mathrm{E} p_{\mathrm{A} / \mathrm{B}}^{\circ}$ to close the recursion for $E p_{A}$. One thus needs to follow the dynamics of the vector Ep $\equiv$ $\left(\mathrm{E} p_{\mathrm{A}}^{\circ}, \mathrm{E} p_{\mathrm{A} / \mathrm{A}}^{\circ}, \mathrm{E} p_{\mathrm{AB}}^{\circ}, \mathrm{E} p_{\mathrm{A} / \mathrm{B}}^{\circ}\right)$. The recursion of the variables evaluated under neutrality over the reproduction phase is

$\mathrm{Ep}_{\mathrm{A} / \mathrm{A}}^{\circ}=\frac{1}{N} \mathrm{E} p_{\mathrm{A}}^{\circ}+\left(\frac{N-1}{N}\right) \mathrm{E} p_{\mathrm{A} / \mathrm{A}}^{\circ}$

$\mathrm{E} p_{\mathrm{AB}}^{\circ}=\mathrm{E} p_{\mathrm{AB}}^{\circ}$

$\mathrm{Ep}_{\mathrm{A} / \mathrm{B}}^{\circ}=\frac{1}{N} \mathrm{E}_{\mathrm{AB}}^{\circ}+\left(\frac{N-1}{N}\right) \mathrm{E}_{\mathrm{A} / \mathrm{B}}^{\circ}$,

and over the recombination phase

$\mathrm{E} p_{\mathrm{A} / \mathrm{A}}^{\circ}=\mathrm{E} p_{\mathrm{A} / \mathrm{A}}^{\circ}$

$\mathrm{E} p_{\mathrm{AB}}^{\circ}{ }^{\prime \prime}=(1-r) \mathrm{E}_{\mathrm{AB}}^{\circ}{ }^{\prime}+r \mathrm{E} p_{\mathrm{A} / \mathrm{B}}^{\circ}$

$\mathrm{E} p_{\mathrm{A} / \mathrm{B}}^{\circ}{ }^{\prime \prime}=\mathrm{E} p_{\mathrm{A} / \mathrm{B}}^{\circ}$.

Note that inserting the last two equations of Eq. (53) into the last two equations of Eq. (54) gives the recursions established previously (Eq. (28)).

These two systems of equations, in combination with Eqs. (51) and (52), can then be used to evaluate the transition matrix $\mathbf{A}^{\cdot}=\mathbf{A}^{\circ}+\delta \dot{\mathbf{A}}$ of Ep for this model. We implemented this as $\mathbf{A}=\mathbf{R S}$, where $\mathbf{S}$ describes the transformation of Ep over the reproduction phase, and $\mathbf{R}$ describes the transformation of Ep over the recombination phase and is not affected by selection; in particular, $\mathbf{A}^{\bullet}=\mathbf{R}^{\circ}\left(\mathbf{S}^{\circ}+\delta \dot{\mathbf{S}}\right)$.

For this model, the reference vector is $\boldsymbol{\wp}=\left(\mathrm{E} p_{\mathrm{A}}^{\cdot}, \mathrm{E} p_{\mathrm{A}}^{\cdot}, \mathrm{E} p_{\mathrm{AB}}^{\circ}\right.$, $\left.\mathrm{E} p_{\mathrm{AB}}^{\circ}\right)$, so that the initial values $\mathbf{v}(0)$ are $\left(0, v_{\mathrm{A} / \mathrm{A}}(0), 0, v_{\mathrm{A} / \mathrm{B}}(0)\right)$, where

$v_{\mathrm{A} / \mathrm{A}}(0)=-\left(p_{\mathrm{A}}(0)-p_{\mathrm{A}}^{2}(0)\right)\left(\frac{N}{N-1}\right)$,

and

$v_{\mathrm{A} / \mathrm{B}}(0)=-\left(p_{\mathrm{AB}}(0)-p_{\mathrm{A}}(0) p_{\mathrm{B}}(0)\right)\left(\frac{N}{N-1}\right)$.

The first-order Taylor polynomial of the fixation probability of the mutant is then given by $\pi_{\dot{A}}=p_{A}(0)+\delta \dot{\pi}_{\mathrm{A}}$ with

$\dot{\pi}_{\mathrm{A}}=-(N-1)\left(s_{\mathrm{A}} v_{\mathrm{A} / \mathrm{A}}(0)+\frac{s_{\mathrm{B}} v_{\mathrm{A} / \mathrm{B}}(0)}{1+r(N-1)}\right)$, which is a decreasing function of the recombination rate.

A single initial copy of the mutant allele appears on a mutant background at locus $B$ with probability $p_{B}(0)$, in which case $v_{\mathrm{A} / \mathrm{A}}(0)=-1 / N$ and $v_{\mathrm{A} / \mathrm{B}}(0)=-\left(1-p_{\mathrm{B}}(0)\right) /(N-1)$. The same copy of the mutant appears on a resident background with probability $1-p_{\mathrm{B}}(0)$, in which case $v_{\mathrm{A} / \mathrm{A}}(0)=-1 / N$ and $v_{\mathrm{A} / \mathrm{B}}(0)=$ $p_{B}(0) /(N-1)$, so that the initial association between mutant alleles is equal to zero. The perturbation of the average fixation probability of a single mutant is

$$
\begin{aligned}
\dot{\bar{\pi}}_{\mathrm{A}}= & p_{\mathrm{B}}(0)\left(\frac{N-1}{N}\right)\left(s_{\mathrm{A}}+\frac{s_{\mathrm{B}} N\left(1-p_{\mathrm{B}}(0)\right)}{[1+r(N-1)](N-1)}\right) \\
& +\left(1-p_{\mathrm{B}}(0)\right)\left(\frac{N-1}{N}\right)\left(s_{\mathrm{A}}-\frac{s_{\mathrm{B}} N p_{\mathrm{B}}(0)}{[1+r(N-1)](N-1)}\right),
\end{aligned}
$$

which simplifies to

$\dot{\bar{\pi}}_{\mathrm{A}}=s_{\mathrm{A}}\left(\frac{N-1}{N}\right)$.

This is precisely the perturbation of the probability of fixation of a single mutant in a one-locus model (e.g. Eq. (40)). We do not yet detect any Hill-Robertson effect (Hill and Robertson, 1966; Barton, 1995; Barton and Otto, 2005), as it should involve at least thirdorder terms of the form $s_{A} s_{B}^{2}$. This is so because interference of locus $B$ on locus A depends on the extent to which the mutant allele at locus $A$ is associated with the mutant at locus $B$ times the selection coefficient at locus $B$, and the effect of selection on the LD must be at least of order $s_{\mathrm{A}} s_{\mathrm{B}}$ to affect its dynamics (Barton and Otto, 2005; Roze, in press, and see below).

\subsubsection{First-order effects on $\pi_{\dot{A}}^{\cdot}$}

In order to evaluate $\pi_{\dot{A B}_{B}}$ we need to compute the recursion for $\mathrm{Ep} p_{\mathrm{AB}}$ and $\mathrm{E} \mathrm{p}_{\mathrm{A} / \mathrm{B}}$. Proceeding as in the last section, we substitute Eq. (47) into Eq. (43), from which we have

$$
\begin{aligned}
\mathrm{E}^{\cdot}\left[p_{\mathrm{AB}}^{\prime} \mid \mathbf{p}\right] & =\mathrm{E}_{i}\left[p_{\mathrm{AB}(i)}+\delta s_{\mathrm{A}}\left(p_{\mathrm{A}(i)}-p_{\mathrm{A}}\right) p_{\mathrm{AB}(i)}+\delta s_{\mathrm{B}}\left(p_{\mathrm{B}(i)}-p_{\mathrm{B}}\right) p_{\mathrm{AB}(i)}\right] \\
& =p_{\mathrm{AB}}+\delta s_{\mathrm{A}}\left(p_{\mathrm{AB}}-p_{\mathrm{A}} p_{\mathrm{AB}}\right)+\delta s_{\mathrm{B}}\left(p_{\mathrm{AB}}-p_{\mathrm{B}} p_{\mathrm{AB}}\right),
\end{aligned}
$$

where we used $p_{\mathrm{A}(i)} p_{\mathrm{AB}(i)}=p_{\mathrm{AB}(i)}$ and $p_{\mathrm{B}(i)} p_{\mathrm{AB}(i)}=p_{\mathrm{AB}(i)}$ to obtain the second line, and integrating the recursion over all sample paths gives

$\mathrm{E} p_{\mathrm{AB}}^{\prime}=\mathrm{E} p_{\mathrm{AB}}^{\cdot}+\delta s_{\mathrm{A}}\left(\mathrm{E}_{\mathrm{AB}}^{\circ}-\mathrm{E}_{\mathrm{A} / \mathrm{AB}}^{\circ}\right)+\delta s_{\mathrm{B}}\left(\mathrm{E}_{\mathrm{AB}}^{\circ}-\mathrm{E}_{\mathrm{B} / \mathrm{AB}}^{\circ}\right)$.

Inserting Eq. (47) into Eq. (44) gives

$$
\begin{aligned}
\mathrm{E} & {\left[p_{\mathrm{A} / \mathrm{B}}^{\prime} \mid \mathbf{p}\right]=\frac{1}{N} \mathrm{E}_{i}\left[p_{\mathrm{AB}(i)}+2 \delta s_{\mathrm{A}}\left(p_{\mathrm{A}(i)}-p_{\mathrm{A}}\right) p_{\mathrm{AB}(i)}+2 \delta s_{\mathrm{B}}\left(p_{\mathrm{B}(i)}-p_{\mathrm{B}}\right) p_{\mathrm{AB}(i)}\right] } \\
& +\left(\frac{N-1}{N}\right) \mathrm{E}_{i, k \neq i}\left[p_{\mathrm{A}(i)} p_{\mathrm{B}(k)}+\delta s_{\mathrm{A}}\left(p_{\mathrm{A}(i)}+p_{\mathrm{A}(k)}-2 p_{\mathrm{A}}\right) p_{\mathrm{A}(i)} p_{\mathrm{B}(k)}\right. \\
& \left.+\delta s_{\mathrm{B}}\left(p_{\mathrm{B}(i)}+p_{\mathrm{B}(k)}-2 p_{\mathrm{B}}\right) p_{\mathrm{A}(i)} p_{\mathrm{B}(k)}\right],
\end{aligned}
$$

and integration over all sample paths produces

$$
\begin{aligned}
& \mathrm{E}_{\mathrm{A} / \mathrm{B}}^{\prime}=\frac{1}{N} \mathrm{E}_{\mathrm{AB}}^{\cdot}+\left(\frac{N-1}{N}\right) \mathrm{E}_{\mathrm{A} / \mathrm{B}}^{\cdot} \\
& +\delta \frac{2}{N}\left(s_{\mathrm{A}}\left[\mathrm{E}_{\mathrm{AB}}^{\circ}-\mathrm{E}_{\mathrm{A} / \mathrm{AB}}^{\circ}\right]+s_{\mathrm{B}}\left[\mathrm{E}_{\mathrm{AB}}^{\circ}-\mathrm{E}_{\mathrm{B} / \mathrm{AB}}^{\circ}\right]\right) \\
& +\delta\left(\frac{N-1}{N}\right)\left(s_{\mathrm{A}}\left[\mathrm{E}_{\mathrm{A} / \mathrm{B}}^{\circ}+\mathrm{E}_{\mathrm{A} / \mathrm{AB}}^{\circ}-2 \mathrm{E}_{\mathrm{A} /(\mathrm{A} / \mathrm{B})}^{\circ}\right]\right. \\
& \left.+s_{\mathrm{B}}\left[\mathrm{E}^{\circ}{ }_{\mathrm{A} / \mathrm{B}}^{\circ}+\mathrm{E} p_{\mathrm{B} / \mathrm{AB}}^{\circ}-2 \mathrm{E}_{\mathrm{B} /(\mathrm{A} / \mathrm{B})}^{\circ}\right]\right),
\end{aligned}
$$

where

$$
\mathrm{Ep}_{V /(\mathrm{A} / \mathrm{B})}^{\circ} \equiv \frac{1}{N} \mathrm{E}_{V \mathrm{~A} / \mathrm{B}}^{\circ}+\frac{1}{N} \mathrm{E}_{V \mathrm{~B} / \mathrm{A}}^{\circ}+\left(\frac{N-2}{N}\right) \mathrm{Ep}_{V / \mathrm{A} / \mathrm{B}}^{\circ} .
$$


In order to close these recursions, we need the additional neutral expectations $\mathrm{E} p_{\mathrm{A} / \mathrm{AB}}^{\circ}, \mathrm{E} p_{\mathrm{B} / \mathrm{AB}}^{\circ}, \mathrm{E} p_{\mathrm{A} / \mathrm{A} / \mathrm{B}}^{\circ}$ and $\mathrm{E} p_{\mathrm{B} / \mathrm{A} / \mathrm{B}}^{\circ}$. For simplicity, we evaluate $\dot{\pi}_{A B}$ only for the case where the double mutant is introduced initially as a single copy in the population, in which case the dynamics of $\mathrm{E} p_{\mathrm{A} / \mathrm{AB}}^{\circ}$ is equivalent to that of $\mathrm{E} p_{\mathrm{B} / \mathrm{AB}}^{\circ}$ and the dynamics of $\mathrm{E} p_{\mathrm{A} / \mathrm{A} / \mathrm{B}}^{\circ}$ is equivalent to that of $\mathrm{E} p_{\mathrm{B} / \mathrm{A} / \mathrm{B}}^{\circ}$. It is then sufficient to consider the dynamics of the vector $\mathrm{Ep} \equiv\left(\mathrm{E} p_{\mathrm{AB}}^{\cdot}, \mathrm{E} p_{\mathrm{A} / \mathrm{B}}^{\circ}, \mathrm{E} p_{\mathrm{A} / \mathrm{AB}}^{\circ}, \mathrm{E} p_{\mathrm{A} / \mathrm{A} / \mathrm{B}}^{\circ}\right)$. We already have the recursions for the first two moments under neutrality (Eqs. (53)-(54)) and the recursions for the two other ones over the reproduction phase is

$\mathrm{Ep}_{\mathrm{A} / \mathrm{AB}}^{\circ}=\frac{1}{N} \mathrm{E}_{\mathrm{AB}}^{\circ}+\left(\frac{N-1}{N}\right) \mathrm{E}_{\mathrm{A} / \mathrm{AB}}^{\circ}$

and

$$
\begin{aligned}
& \mathrm{E} p_{\mathrm{A} / \mathrm{A} / \mathrm{B}}^{\circ}=\frac{1}{N^{2}} \mathrm{E} p_{\mathrm{AB}}^{\circ}+\left(\frac{N-1}{N^{2}}\right) E p_{\mathrm{A} / \mathrm{B}}^{\circ} \\
& \quad+2\left(\frac{N-1}{N^{2}}\right) \mathrm{E} p_{\mathrm{A} / \mathrm{AB}}^{\circ}+\left(\frac{(N-1)(N-2)}{N^{2}}\right) \mathrm{E} p_{\mathrm{A} / \mathrm{A} / \mathrm{B}}^{\circ}
\end{aligned}
$$

Over the recombination phase we have

$\mathrm{E}_{\mathrm{A} / \mathrm{AB}}^{\circ}{ }^{\prime \prime}=(1-r) \mathrm{E}_{\mathrm{A} / \mathrm{AB}}^{\circ}+r \mathrm{E}_{\mathrm{A} / \mathrm{B} / \mathrm{A}^{\prime}}^{\circ}$

$\mathrm{E} p_{\mathrm{A} / \mathrm{A} / \mathrm{B}}^{\circ}=\mathrm{E} p_{\mathrm{A} / \mathrm{A} / \mathrm{B}}^{\circ}$.

From the above equations, we find that

$\pi_{\mathrm{AB}}^{\circ}=\frac{1}{N}-\left(\frac{N-1}{N}\right)\left(\frac{r}{1+r(N-1)}\right)$,

which is Eq. (13) with $D_{\mathrm{AB}}(0)=1 / N$. The first-order perturbation of the fixation probability of a double mutant is

$\dot{\pi}_{\mathrm{AB}}=\left(\frac{N-1}{N}\right)\left(\frac{s_{\mathrm{A}}+s_{\mathrm{B}}}{1+r(N-1)}\right)\left(\frac{3 N-2+r(2+N(N-2))}{3 N-2+r(2+N(N-3))}\right)$,

where the last ratio is approximately equal to one, and with this approximation our result becomes equivalent to the one established from consideration of the diffusion operator by Ohta (1968, eq. 8).

\subsubsection{Second-order effects on $\pi_{\mathrm{A}}$}

In order to evaluate the second-order effects of selection on $\pi_{\mathrm{A}}$, we have to insert the second-order polynomial

$$
\begin{aligned}
w_{i}^{*}= & 1+\delta s_{\mathrm{A}}\left(p_{\mathrm{A}(i)}-p_{\mathrm{A}}\right)+\delta s_{\mathrm{B}}\left(p_{\mathrm{B}(i)}-p_{\mathrm{B}}\right) \\
& +\delta^{2} s_{\mathrm{A}} s_{\mathrm{B}}\left(p_{\mathrm{A}(i)} p_{\mathrm{B}(i)}-p_{\mathrm{AB}}\right)-\delta^{2}\left(s_{\mathrm{A}} p_{\mathrm{A}}+s_{\mathrm{B}} p_{\mathrm{B}}\right) \\
& \times\left[s_{\mathrm{A}}\left(p_{\mathrm{A}(i)}-p_{\mathrm{A}}\right)+s_{\mathrm{B}}\left(p_{\mathrm{B}(i)}-p_{\mathrm{B}}\right)\right]
\end{aligned}
$$

of the fitness function Eq. (46) into Eq. (43). Integrating over all sample paths yields

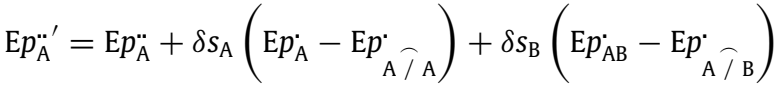

$$
\begin{aligned}
& +\delta^{2} s_{\mathrm{A}} s_{\mathrm{B}}\left(\mathrm{E}_{\mathrm{AB}}^{\circ}-\mathrm{E}_{\mathrm{A} / \mathrm{AB}}^{\circ}\right)-\delta^{2} s_{\mathrm{A}}^{2}\left(\mathrm{E}_{\mathrm{A} / \mathrm{A}}^{\circ}-\mathrm{Ep}_{\mathrm{A} / \mathrm{A} / \mathrm{A}}^{\circ} \widehat{C}^{\circ}\right) \\
& -\delta^{2} s_{\mathrm{A}} s_{\mathrm{B}}\left(\mathrm{E}_{\mathrm{A} / \mathrm{B}}^{\circ}+\mathrm{E}_{\mathrm{A} / \mathrm{AB}}^{\circ}-2 \mathrm{E} p_{\mathrm{A} / \mathrm{A} / \mathrm{B}}^{\circ}\right) \\
& -\delta^{2} s_{\mathrm{B}}^{2}\left(\mathrm{Ep}_{\mathrm{B} / \mathrm{AB}}^{\circ}-\mathrm{Ep}_{\mathrm{A} / \mathrm{B} / \mathrm{B}}^{\circ}\right),
\end{aligned}
$$

where

$$
\begin{aligned}
\mathrm{Ep}_{U / V T W}^{\circ} \equiv & \frac{1}{N^{2}} \mathrm{E}_{U V W}^{\circ}+\left(\frac{N-1}{N^{2}}\right) \mathrm{Ep}_{U / V W}^{\circ} \\
& +\left(\frac{N-1}{N^{2}}\right) \mathrm{Ep}_{U V / W}^{\circ}+\left(\frac{N-1}{N^{2}}\right) \mathrm{Ep}_{U W / V}^{\circ} \\
& +\left(\frac{(N-1)(N-2)}{N^{2}}\right) \mathrm{Ep}_{U / V / W}^{\circ}
\end{aligned}
$$

involves sampling sets of loci from three individuals with replacement. These equations illustrate that calculations performed by hand become rapidly tedious. In the sequel of this paper, therefore, we present perturbation results calculated directly with Mathematica.

We obtain that the second-order perturbation of the average fixation probability of the mutant allele is

$$
\ddot{\bar{\pi}}_{\mathrm{A}}=s_{\mathrm{A}}^{2}\left(\frac{N-1}{N}\right)\left(\frac{N(2 N-7)+4}{3 N-2}\right),
$$

and as expected we do not yet detect any Hill-Robertson effect.

\subsubsection{Third-order effects on $\pi_{\mathrm{A}}$}

In order to evaluate the third-order perturbation of $\pi_{\mathrm{A}}$, we need to calculate the dynamics of $E p_{A}$ from the third-order expansion of $w_{i}$ (Eq. (46)). The resulting recursion and those of the associated moments are complicated. Taking a large size approximation (large $N$ as in the diffusion limit) did not simplify them enough to gain a better intuitive understanding of the possible interference of locus B on locus A. Nevertheless, in order to gain such an understanding we turn to the following considerations. The mutant alleles at both loci, $\mathrm{A}$ and $\mathrm{B}$, provide a fitness advantage to their carriers, and the extent to which selection on the mutant at locus B is likely to spill over onto the change of gene frequency at locus A depends on the LD between the two alleles. This can be seen by inserting Eq. (46) into Eq. (43), and expressing $\Delta \mathrm{E} p_{\mathrm{A}}=\mathrm{E}\left[\mathrm{E}\left[p_{\mathrm{A}}^{\prime} \mid \mathbf{p}\right]-p_{\mathrm{A}}\right]$ as

$$
\begin{aligned}
\Delta \mathrm{E} p_{\mathrm{A}} & =\mathrm{E}\left[\frac{\delta s_{\mathrm{A}} p_{\mathrm{A}}\left(1-p_{\mathrm{A}}\right)}{1+\delta s_{\mathrm{A}} p_{\mathrm{A}}}\right] \\
& +\mathrm{E}\left[\frac{\left(p_{\mathrm{AB}}-p_{\mathrm{A}} p_{\mathrm{B}}\right)\left(\delta s_{\mathrm{B}}+\delta^{2} s_{\mathrm{A}} s_{\mathrm{B}}\right)}{\left(1+\delta s_{\mathrm{A}} p_{\mathrm{A}}\right)\left(1+\delta s_{\mathrm{A}} p_{\mathrm{A}}+\delta s_{\mathrm{B}} p_{\mathrm{B}}+\delta^{2} s_{\mathrm{A}} s_{\mathrm{B}} p_{\mathrm{AB}}\right)}\right] .
\end{aligned}
$$

This equation illustrates that if the $\mathrm{LD}$ defined as $p_{\mathrm{AB}}-p_{\mathrm{A}} p_{\mathrm{B}}$ is positive (LD calculated by sampling loci from different individuals with replacement $p_{\mathrm{A}} p_{\mathrm{B}}=p_{\mathrm{A} / \mathrm{B}}$ ), selection at locus $\mathrm{B}$ will facilitate the spread of the mutant at locus $A$, while if the LD is negative, interference between loci occurs.

The initial LD, averaged over the two backgrounds in which a mutant may appear, is equal to zero (see section "First-order effects on $\pi_{\mathrm{A}}$ "). For interference to occur, the interaction between selection and drift must thus lead to the built-up of an LD such that the average (over the two backgrounds) of the second expectation in Eq. (74) is negative. As the random variable in Eq. (74) is not linear in $p_{\mathrm{AB}}$, its expectation may depend on all the moments of the LD distribution. A third-order Taylor expansion in the selection strength gives

$$
\begin{aligned}
E\left[\frac{\left(p_{\mathrm{AB}}-p_{\mathrm{A}} p_{\mathrm{B}}\right)\left(\delta s_{\mathrm{B}}+\delta^{2} s_{\mathrm{A}} s_{\mathrm{B}}\right)}{\left(1+\delta s_{\mathrm{A}} p_{\mathrm{A}}\right)\left(1+\delta s_{\mathrm{A}} p_{\mathrm{A}}+\delta s_{\mathrm{B}} p_{\mathrm{B}}+\delta^{2} s_{\mathrm{A}} s_{\mathrm{B}} p_{\mathrm{AB}}\right)}\right] \\
=\left(\delta s_{\mathrm{B}}+\delta^{2} s_{\mathrm{A}} s_{\mathrm{B}}\right) \mathrm{E}\left[p_{\mathrm{AB}}-p_{\mathrm{A}} p_{\mathrm{B}}\right] \\
\quad-\delta^{3} s_{\mathrm{A}} s_{\mathrm{B}}^{2} \mathrm{E}\left[\left(p_{\mathrm{AB}}-p_{\mathrm{A}} p_{\mathrm{B}}\right)^{2}\right]-\delta s_{\mathrm{B}}\left(1+\delta s_{\mathrm{A}}\right) \\
\quad \times \mathrm{E}\left[\left(p_{\mathrm{AB}}-p_{\mathrm{A}} p_{\mathrm{B}}\right)\left(2 \delta s_{\mathrm{A}} p_{\mathrm{A}}+\delta s_{\mathrm{B}} p_{\mathrm{B}}\right)\right]+O\left(\frac{\delta^{3}}{N^{2}}\right) .
\end{aligned}
$$


The first term on the right-hand side reflects the increase in the selective pressure on the mutant at locus A due to increased fecundity to its carrier when the allele is associated to the mutant at locus $B$, while the second and third terms reflect the increase in competition felt by the carriers of the mutant allele at locus A when its is associated to the other mutant allele. The first competition term depends on the second moment $\mathrm{E}\left[\left(p_{\mathrm{AB}}-p_{\mathrm{A}} p_{\mathrm{B}}\right)^{2}\right]$ of $\mathrm{LD}$ (whose average over the two backgrounds in which the mutant may initially appear can be thought of as the variance of LD) and is always positive, even in the neutral case. The expectation in the second competition term will built up if the covariances between $L D$ and the beneficial alleles build up, which will occur because mutant allele frequencies increases more rapidly when there is a positive LD (Barton and Otto, 2005, p. 2357, we also mention that the terms in Eq. (75) agree qualitatively with those in eq. 4a obtained by Barton and Otto by perturbing the deterministic regime).

Eq. (75) shows that if the average of the expected LD is negative, interference occurs regardless of the parameter values. The initial variance in LD does indeed lead to a negative average expected LD in the presence of selection (Barton and Otto, 2005; Roze, in press), and in order to see this under our life-cycle assumptions, it is useful to express the LD as

$$
\begin{aligned}
\mathrm{E}\left[p_{\mathrm{AB}}^{\prime}-p_{\mathrm{A}}^{\prime} p_{\mathrm{B}}^{\prime}\right] & =\left(\frac{N-1}{N}\right) \mathrm{E}\left[p_{\mathrm{AB}}^{\prime}-p_{\mathrm{A} / \mathrm{B}}^{\prime}\right] \\
& =\left(\frac{N-1}{N}\right) \mathrm{E}\left[\mathrm{E}\left[p_{\mathrm{AB}}^{\prime} \mid \mathbf{p}\right]-\mathrm{E}\left[p_{\mathrm{A} / \mathrm{B}}^{\prime} \mid \mathbf{p}\right]\right],
\end{aligned}
$$

where, on substitution of Eqs. (43) and (44), we obtain after rearrangements

$$
\begin{aligned}
\mathrm{E} & \left.p_{\mathrm{AB}}^{\prime}-p_{\mathrm{A}}^{\prime} p_{\mathrm{B}}^{\prime}\right]=\left(\frac{N-1}{N}\right) \\
& \times \mathrm{E}[\underbrace{\left[\frac{N-1}{N}\right)\left(\mathrm{E}_{i}\left[w_{i} p_{\mathrm{AB}(i)}\right]-\mathrm{E}_{i, k \neq i}\left[w_{i} w_{k} p_{\mathrm{A}(i)} p_{\mathrm{B}(k)}\right]\right)}_{\mathrm{LD} \text { after selection }} \\
& \left.-\frac{1}{N} \mathrm{E}_{i}\left[w_{i}\left(w_{i}-1\right) p_{\mathrm{AB}(i)}\right]\right] .
\end{aligned}
$$

Under our multiplicative selection scheme, the LD after selection is the same as that before selection. Hence, if the initial average LD is zero, the average of the first term in Eq. (77) is zero. The second term in Eq. (77) is the increase in coalescence probability resulting from selection acting on gamete $A B$. The effect of this increase in coalescence probability on $\mathrm{LD}$ is zero when the mutants initially reside on two different gametes $\left(p_{\mathrm{AB}(i)}=0\right)$, and thus when the initial LD is negative. This illustrates the notions that selection does not act symmetrically on the two different initial LDs created by the appearance of a mutant allele on a polymorphic background (Barton and Otto, 2005), and, consequently, that a negative average expected LD will build up under second-order effects of selection (Barton and Otto, 2005; Roze, in press; under first-order effects we have $\left.\mathrm{E}_{j}^{:}\left[w_{i}\left(w_{i}-1\right) p_{\mathrm{AB}(i)}\right]=0\right)$. This shows that the average change of mutant frequency at locus $A$ is decreased by the presence of the mutant allele segregating at locus B; namely, interference between alleles occurs, and the Hill-Robertson effect kicks in.

In order to quantify the reduction in the fixation probability due to interference, we computed $\Delta \pi_{\mathrm{HR}}$, the average third-order perturbation of $\bar{\pi}_{\mathrm{A}}$ minus the same quantity with $s_{\mathrm{B}}$ set to zero. The resulting equation is complicated and its explicit expression is presented in the Appendix (Eq. (A.34)). In the absence of recombination ( $r=0$ in Eq. (A.34)), the reduction of the fixation

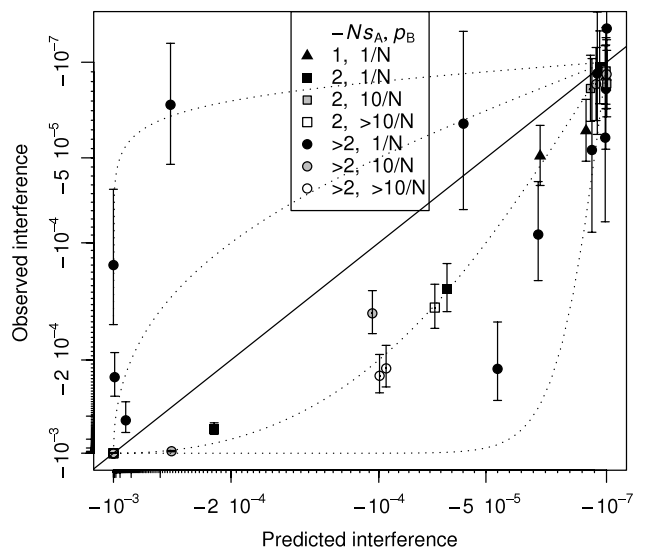

Fig. 1. Hill-Robertson interference was estimated in simulations and compared to predictions (Eq. (A.34) of the Appendix) for 49 combinations of model parameters $\left(s_{\mathrm{A}}=s_{\mathrm{B}}, N s_{\mathrm{A}}=1\right.$ to $500, r=0.001$ to $0.1, N=100$ to $10000, p_{\mathrm{B}}(0)=1 / \mathrm{N}$ to $1 / 2$ ). The range of interference values considered led us to use an inverse logistic scale $\left(z \rightarrow e^{f z} /\left(1+e^{f z}\right)\right.$, with $\left.f=10000\right)$ on both axes. Error bars are \pm 2 standard errors. The dotted lines show twofold and tenfold over- and under-prediction of the observed interferences. Greater that twofold prediction errors therefore occur mostly for $N s_{\mathrm{A}}>2$ (circled-shaped symbols). The two top-left outliers have $N s_{\mathrm{A}}=$ 200 and 500, and $r=0.001$.

probability is given by

$$
\begin{aligned}
\Delta \pi_{\mathrm{HR}}= & -s_{\mathrm{A}} s_{\mathrm{B}}^{2} p_{\mathrm{B}}(0)\left(1-p_{\mathrm{B}}(0)\right) N^{2} \\
& \times\left(\frac{36 N^{3}-81 N^{2}+66 N-20}{108 N^{3}-270 N^{2}+240 N-72}\right),
\end{aligned}
$$

where the ratio is approximatively equal to $1 / 3$ when $N$ becomes large. In the presence of recombination, another approximation can be obtained by assuming large population size, in which case we have $\Delta \pi_{\mathrm{HR}}=-s_{\mathrm{A}} s_{\mathrm{B}}^{2} p_{\mathrm{B}}(0) /\left(2 r^{2}\right)+O\left(1 / N^{2}\right)$, where we also assumed that $p_{\mathrm{B}}(0) \ll 1$. This result does not imply that the perturbation approach is valid only for $N r>1$, because the exact third-order result for $\Delta \pi_{\mathrm{HR}}$ (Eq. (A.34)) is valid for any combination of values of $r$ and $N$ (i.e., no singularity when $r \rightarrow 0$ for all $N$ ). The perturbation approach does, therefore, not make any assumption on the value $r$ should take given that of $N$.

In Figs. 1 and 2, we compare the exact third-order result for $\Delta \pi_{\mathrm{HR}}$ (Eq. (A.34)) with those obtained from simulations for $s_{\mathrm{A}}=$ $s_{\mathrm{B}}$ and $s_{\mathrm{B}}=10 s_{\mathrm{A}}$, respectively. When $N s_{\mathrm{B}} \leq 5$, the observed fixation probability departs from the predicted one as the latter increases (which here corresponds not only to relatively large $s_{\mathrm{B}}$ but also large initial value, $p_{\mathrm{B}}=0.5$, of the preexisting segregating allele when the mutant appears). When $N s_{\mathrm{B}}>5$, relatively large discrepancies can be observed when the predicted values are low, in particular in large populations. Hence, the perturbation approach is valid for weak selection regimes only, and involves assumptions on the values $\delta$ can take given that of $N$.

The limitation to the weak selection regime of the perturbation approach can be understood from two considerations. First, an even Taylor polynomial in a selection coefficient $s$ will give qualitatively equivalent answers for strong large positive and large negative $s$, which is not the desired answer in general. Second, in one-locus models (and surely in many multilocus models), large $N s$ matters more than large $s$, and thus for small $|s|$ but large $|N s|$ Taylor polynomials will give poor approximations. This can be illustrated by the well-known diffusion approximation for the fixation probability of a single mutant allele with selective coefficient $s$ over the resident, which is

$\pi=\frac{1-e^{-2 s}}{1-e^{-2 N s}}$ 


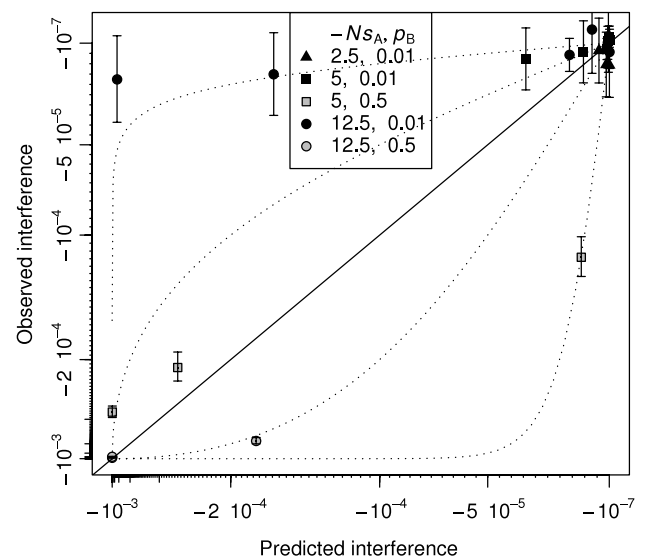

Fig. 2. Same as in Fig. 1 except that $s_{\mathrm{A}}=10 s_{\mathrm{B}}, N=100$, and $N s_{\mathrm{A}}$ and $p_{\mathrm{B}}(0)$ values as given in the box of the graph (21 parameter combinations). The top-left outlier has $r=0.001$.

which illustrates that the fixation probability depends on the Ns product (Crow and Kimura, 1970; Gillespie, 2004; Ewens, 2004). As pointed out by Hill (1972), Taylor polynomial expansions in powers of $s$ of this expression are ill-behaved for large Ns values. For example the polynomials of degree 4 of Eq. (79) in $s$ is

$$
\begin{aligned}
\frac{1}{N} & +\frac{s(N-1)}{N}+\frac{s^{2}((N-3) N+2)}{3 N}-\frac{s^{3}(N-1)^{2}}{3 N} \\
& -\frac{s^{4}\left(N\left(N^{3}-10 N+15\right)-6\right)}{45 N},
\end{aligned}
$$

which turns out to be negative for $s>0.05$ when $N=100$; the same results hold for even-order polynomials of degree $\geq 4$. Thus the perturbation approximation of the diffusion result works only for low Ns. Polynomials of order up to 100 are easy to evaluate using the Mathematica implementation of the above algorithms, so it can be checked that the general perturbation method presented here yields expansions for the one-locus model that are very close to expansions of the diffusion approximation. For instance, the first-order perturbation in Eq. (80) agrees with Eq. (40), the secondorder perturbation in Eq. (80) is numerically close to Eq. (73), and the fourth-order perturbations become equivalent for large $N$. Thus, the general method works only for low $N \delta$ values essentially because the fixation probabilities depend also on $N \delta$ so that they are poorly approximated by Taylor polynomials in $\delta$, which can be thought of as the largest of the selection coefficient in the multilocus setting.

\section{Discussion}

We have presented a formalism to evaluate $n$ th-order perturbation expansions of moments of allelic states in finite populations of constant size without class structure under arbitrary strength of selection $\delta$ and gene action. This covers epistatic interactions within genomes as well as synergistic interactions between them. This formalism should apply unchanged to geographically structured populations, although the dimension of the matrices to be considered could become prohibitive. Taking a diffusion limit (large $N$, small $\delta$ ) to simplify the recursions may be useful in some cases. In the absence of mutation, the long-term limit of the moments provide multilocus fixation probabilities. Our analysis shows that, regardless of the strength of selection, fixation probabilities can be expressed in terms of selection coefficients weighted by mean first passage times. As detailed in the section "Results", these relationships generalize the one-locus result, where the mean first passage times are mean coalescence times, which can themselves be expressed in terms of relatedness coefficients (Slatkin, 1991), thereby providing a link to concepts of inclusive fitness theory (Hamilton, 1964, 1970).

We have also illustrated how to evaluate the recursions necessary to compute explicitly the moments of allelic states under a Wright-Fisher random union of gametes model. This allowed us to apply the approach to a two-locus model with multiplicative effects on fitness in order to obtain analytical expressions for the Hill-Robertson effect under small population size. The Hill-Robertson effect depends on interactions between loci, and it involves at least third-order perturbations of selection on gene frequency change (e.g., Eq. (78)). Comparison of the perturbation method with the results from simulations show that the analytical results give good predictions of the fixation probabilities for only low $N \delta$ values (practically, $N \delta \leqslant 1$ ). In other words, the perturbation approximation works only under weak selection regimes.

By contrast, Otto and Barton (1997) have developed approximations of the Hill-Robertson effect that are valid specifically for stronger selection and very large population size. The limitation of the perturbation analysis follow from the fact that fixation probabilities depend on the product $N \delta$ in the one-locus case (Eq. (79)), and are thus not well approximated in expansions in $\delta$ when $N \delta>1$. Our analysis of the Hill-Robertson effect shows that this carries over to the two-locus case, and the same is likely to hold in a general multilocus context. Thus, unless complemented by other results, the perturbation method yields mainly qualitative results, whose quantitative relevance must be checked on a caseby-case basis.

Nevertheless, one-locus first-order perturbations of the effect of selection on allele frequency change in deterministic models give excellent prediction for long-term phenotypic evolution under a wide spectrum of biological applications involving local genetic drift (as occurs in the infinite island model) with weak assumptions about the frequency of different types of mutations (e.g., Geritz et al., 1998; Pen, 2000; Le Galliard et al., 2003; Leturque and Rousset, 2002; Guillaume and Perrin, 2006). Local genetic drift in infinite structured populations (or global genetic drift in finite panmictic populations) allows populations to cross invasion thresholds (i.e., peak shift or stochastic tunneling) that would not be possible if the local (or total) population was of infinite size. In one-locus models, first-order perturbation of gene frequency change (or of fixation probabilities) predict well the direction of selection when invasion thresholds can be crossed by genetic drift. This is exemplified by the application of Hamilton's rule to the evolution of altruistic helping and dispersal in spatially structured population, where the relatedness quantifies the fluctuations in allele frequency among demes due to local genetic drift under neutrality (e.g., Hamilton and May, 1977; Taylor, 1992; Pen, 2000; Rousset, 2004; Guillaume and Perrin, 2006).

One-locus first-order perturbations are, therefore, indicative of the direction of selection on a mutant allele when genetic drift plays a crucial role in the evolutionary dynamics. Do multilocus first-order perturbations of fixation probabilities predict well the direction of selection in that case? The situation becomes more complicated in two-locus models, where the interaction between loci is likely to depend on the relative magnitude of population size and recombination rate. We compared analytical results of two-locus two-alleles models involving social interactions such as the coevolution of helping and punishment following the strongreciprocity protocol (see Eqs. (A.37)-(A.44), and further examples in prep.), with those of simulations, and our results suggest that first-order perturbation seem to predict well the direction of selection in a multilocus context, unless selection becomes very strong (Fig. 3). 

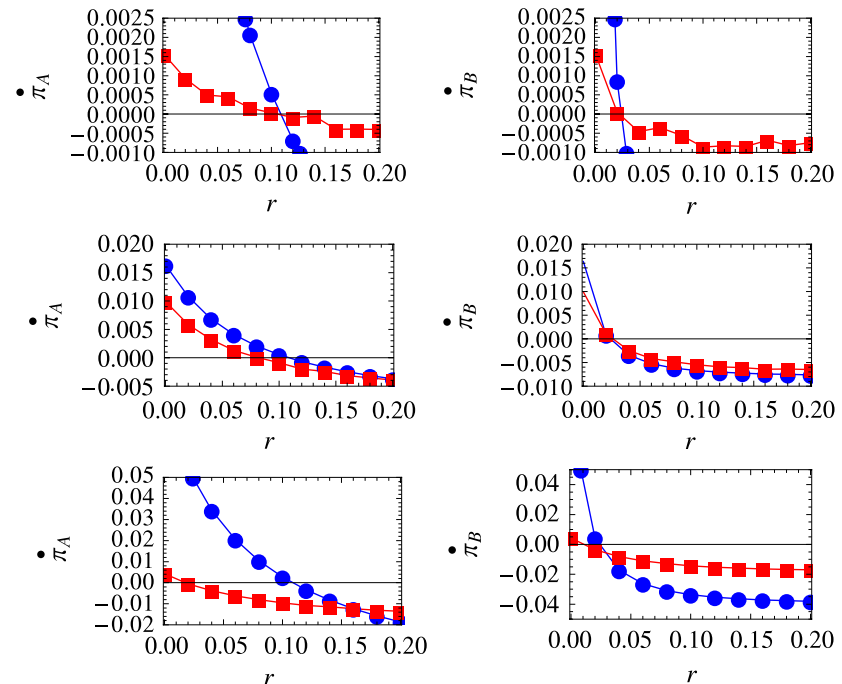

Fig. 3. Comparison of the change in the fixation probabilities $\dot{\pi}_{\mathrm{A}}$ and $\dot{\pi}_{\mathrm{B}}$ for helping and punishment predicted by the analytical model (Eqs. (A.41) and (A.43), dots in the figure) with that observed from simulations (squares, for $10^{6}$ trials), for the case where a single mutant helping allele appears simultaneously with a single punishment allele (i.e., $N-1$ individuals in the population bear the residents alleles at both loci). The panels of the left column stem for the change in the fixation probability of the helping allele, while the panels on the right column stem for that of the punishment allele. Each row of panels gives the change in fixation probabilities under different selection strengths as a function of the recombination rate. For all panels we have $N=50$, and the remaining parameter values are $C_{\mathrm{H}}=0.001, C_{\mathrm{P}}=0.001, B_{\mathrm{H}}=0.005$, and $D_{\mathrm{P}}=0.01$ for the first row of panels; $C_{\mathrm{H}}=0.01, C_{\mathrm{P}}=0.01, B_{\mathrm{H}}=0.05$, and $D_{\mathrm{P}}=0.1$ for the second row; and $C_{\mathrm{H}}=0.05$, $C_{\mathrm{P}}=0.05, B_{\mathrm{H}}=0.25$, and $D_{\mathrm{P}}=0.5$ for the last row.

In the case of the coevolution of helping and punishment firstorder perturbations show that the fixation probabilities of both a mutant helping and a mutant punishment allele introduced initially on the same gamete (i.e., $N-1$ individuals in the population bear the residents alleles at both loci) is greatly increased when the population size is decreased provided the recombination rate is weak (Eqs. (A.41) and (A.43)). However, the perturbations of fixation probabilities also reveal that once it is taken into account that a mutant helping allele may appear on different backgrounds (either a mutant or a resident background at the punishment locus depending on the frequency of the punishment allele), the average perturbation of fixation probability of a helping allele does no longer depend on the recombination rate, and it decreases as population size decreases (Eq. (A.42)). This suggests that, holding everything else constant, finite population size may actually inhibit rather than favor the evolution of helping under the strong-reciprocity protocol. More generally, first-order perturbation may allow us to predict longterm phenotypic evolution in a multilocus context by taking into account the effect of the finiteness of populations.

In summary our analysis has yielded two results. First, we have shown how to evaluate perturbation expansions for moments of allelic states under arbitrary strength of selection and gene action, and express multilocus fixation probabilities in terms of first passage times; that is, in terms of genealogical ties. Second, we have highlighted some shortcomings and advantages of using perturbation expansions to approximate fixation probabilities. This is relevant for further theoretical developments as the evaluation of such approximations has attracted a lot of attention in the context of one-locus frequency-dependent selection (e.g., Wild and Taylor, 2004; Ohtsuki and Nowak, 2006; Lessard and Ladret, 2007b; Grafen, 2007; Kurokawa and Ihara, 2009), and the inclusion of social evolution into multilocus models with genetic drift remains to be explored.

\section{Acknowledgments}

We thank D. Weissman for helpful discussions and J. Van Cleve, P. Fontanillas, D. Roze and the reviewers for helpful comments on the manuscript. LL is supported by a grant from the Swiss NSF and by NIH grant GM28016 to M. W. Feldman. This is publication ISEM 09-049. Simulations were run on the ISEM Cluster.

\section{Appendix}

\section{A.1. Recursion for $\mathbf{v}(t)$}

In this Appendix we show how to define directly from $\mathbf{A}$ reference values $\wp_{S}(t)$, and a vector $\mathbf{v}(t) \equiv\left(v_{S}(t)\right)_{S \in S} \equiv\left(E p_{S}(t)-\right.$ $\left.\wp_{S}(t)\right)$ of centered variables whose elements vanish geometrically fast for large $t$. This allows us to evaluate $\mathbf{d} \equiv \sum_{t=0}^{\infty} \mathbf{v}(t)$ by matrix inversion. For ease of presentation we start with the neutral case, and then generalize to any order of approximation.

\section{A.1.1. The neutral case}

Under neutrality, the coefficient determining the dynamics of each moment of allelic state can be gathered in a transition matrix $\mathbf{A}^{\circ}$ such that the vector $\mathrm{Ep}^{\circ}(t)$ consisting uniquely of neutral moments of allelic state satisfies

$\mathrm{Ep}^{\circ}(t+1)=\mathbf{A}^{\circ} \mathrm{Ep}^{\circ}(t)$.

For instance, for a two-locus model we may need to evaluate the dynamics of $E p_{A / A}^{\circ}(t)$ and that of $E p_{A B}^{\circ}(t)$. In this case, we have to consider $\mathrm{Ep}^{\circ}(t) \equiv\left(\mathrm{Ep}_{\mathrm{A}}^{\circ}(t), \mathrm{E}_{\mathrm{A} / \mathrm{A}}^{\circ}(t), \mathrm{Ep}_{\mathrm{AB}}^{\circ}(t), \mathrm{E}_{\mathrm{A} / \mathrm{B}}^{\circ}(t)\right)$, whose dynamics is described by the matrix

$\mathbf{A}^{\circ}=\left(\begin{array}{cccc}1 & 0 & 0 & 0 \\ a_{1}^{\circ} & 1-a_{1}^{\circ} & 0 & 0 \\ 0 & 0 & a_{2}^{\circ} & 1-a_{2}^{\circ} \\ 0 & 0 & a_{3}^{\circ} & 1-a_{3}^{\circ}\end{array}\right)$,

where $a_{1}^{\circ}$ is the probability that two genes, sampled from homologous loci in two distinct individuals, descend from the same individual in the previous generation; $a_{2}^{\circ}$ is the probability that two genes, sampled from distinct loci from the same individual, descend from the same individual in the previous generation and $a_{3}^{\circ}$ is the probability that two genes, sampled from distinct loci from distinct individuals, descend from the same individual in the previous generation.

In order to obtain a recursion for $\mathbf{v}^{\circ}(t) \equiv\left(v_{Z}^{\circ}(t)\right)_{Z \in \mathcal{Z}} \equiv$ $\left(E p_{Z}^{\circ}(t)-\wp_{Z}^{\circ}(t)\right)$, where $\sum_{t=0}^{\infty}\left(E p_{Z}^{\circ}(t)-\wp_{Z}^{\circ}(t)\right)$ is finite, it suffices to choose the $\wp_{Z}^{\circ}(t)$ 's so that every element of $\mathbf{v}^{\circ}(t)$ vanishes geometrically fast for large $t$. In particular for any set $Z$ of loci sampled from the same or different individuals, $p_{Z}^{\circ}(t)$ and $\wp_{Z}^{\circ}(t)$ must converge to the same value for large $t$, when polymorphism is lost at the set of loci involved in $Z$.

As noted in the main text and illustrated by Eq. (A.2), the $\mathbf{A}^{\circ}$ matrix is block diagonal, with one block for each set of loci. We thus consider one reference value for each block. This reference value could be chosen as any of the $\mathrm{Ep}_{V}^{\circ}(t)$ variable involved in the block, and for definiteness we choose it to be the variable standing for gene copies all taken in the same individual (i.e., $\mathrm{E} p_{\mathrm{A}}^{\circ}(t)$ and $\mathrm{E} p_{\mathrm{AB}}^{\circ}(t)$ for the two blocks in the above example). Formally, a reference vector can be defined as

$\wp^{\circ}(t) \equiv\left(\wp_{Z}^{\circ}(t)\right)_{Z \in \mathcal{Z}} \equiv \sum_{R \in \mathcal{R}} \mathbf{e}_{R} E p_{R}^{\circ}(t)$,

where $\mathcal{R}$ is the set of all reference values, one for each independent neutral systems appearing in $\mathbf{A}^{\circ}$; that is, one for each block. Each $\mathbf{e}_{R}$ is a right eigenvector of one block, associated with a unit eigenvalue of the regular Markov chain $\mathbf{A}^{\circ}$, and augmented with 
zero on rows corresponding to the other blocks. For Eq. (A.2), we have $\mathcal{R}=\{\mathrm{A}, \mathrm{AB}\}$ with corresponding eigenvectors $\mathbf{e}_{\mathrm{A}}=$ $(1,1,0,0)$ and $\mathbf{e}_{\mathrm{AB}}=(0,0,1,1)$, which produces the reference vector $\wp^{\circ}(t)=\left(\mathrm{Ep}_{\mathrm{A}}^{\circ}(t), \mathrm{Ep}_{\mathrm{A}}^{\circ}(t), \mathrm{Ep}_{\mathrm{AB}}^{\circ}(t), \mathrm{E} p_{\mathrm{AB}}^{\circ}(t)\right)$.

With this definition of $\wp^{\circ}(t)$, we will show that $\mathbf{v}^{\circ}(t) \equiv$ $E \mathbf{p}^{\circ}(t)-\wp^{\circ}(t)$ vanishes for large $t$. To achieve our construction we also need a matrix $\mathbf{A}_{\wp}^{\circ}$ such that

$\mathbf{v}^{\circ}(t+1)=\left(\mathbf{A}^{\circ}-\mathbf{A}_{\wp}^{\circ}\right) \mathbf{v}^{\circ}(t)$,

where all eigenvalues of $\mathbf{A}^{\circ}-\mathbf{A}_{\wp}^{\circ}$ are $<1$ in modulus, so that

$$
\begin{aligned}
\mathbf{d}^{\circ} & \equiv \sum_{t=0}^{\infty} \mathbf{v}^{\circ}(t)=\sum_{t=0}^{\infty}\left(\mathbf{A}^{\circ}-\mathbf{A}_{\wp}^{\circ}\right)^{t} \mathbf{v}(0) \\
& =\left(\mathbf{I}-\mathbf{A}^{\circ}+\mathbf{A}_{\wp}^{\circ}\right)^{-1} \mathbf{v}^{\circ}(0)
\end{aligned}
$$

is bounded, and where $\mathbf{v}^{\circ}(0)$ is the initial vector of centered variables.

If Eq. (A.4) holds, then

$$
\mathrm{Ep}^{\circ}(t+1)-\wp^{\circ}(t+1)=\left(\mathbf{A}^{\circ}-\mathbf{A}_{\wp}^{\circ}\right)\left(\mathrm{Ep}^{\circ}(t)-\wp^{\circ}(t)\right),
$$

which gives

$$
\left(\mathbf{A}^{\circ}-\mathbf{A}_{\wp}^{\circ}\right) \boldsymbol{\wp}^{\circ}(t)=\wp^{\circ}(t+1)-\mathbf{A}_{\wp}^{\circ} \mathrm{Ep}^{\circ}(t) .
$$

We define $\mathbf{A}_{\wp}^{\circ}$ so that both sides of this equation are null. First consider the left-hand side. By definition, $\wp^{\circ}(t)$ belongs to the unit-eigenvalue eigenspace of $\mathbf{A}^{\circ}$, i.e., $\mathbf{A}^{\circ} \boldsymbol{\wp}^{\circ}(t)=\boldsymbol{\wp}^{\circ}(t)$, and thus we need to define $\mathbf{A}_{\wp}^{\circ}$ such that $\mathbf{A}_{\wp}^{\circ} \wp^{\circ}(t)=\wp^{\circ}(t)$. It suffices to define $\mathbf{A}_{\wp}^{\circ}$ as the matrix where all rows involved in any given block of equations are replaced by that row of the given block which describes the recursion for the reference value for this block (which we have taken to be the variable in this block standing for gene copies all taken in the same individual). For the example given above Eq. (A.2), this gives

$\mathbf{A}_{\wp}^{\circ}=\left(\begin{array}{cccc}1 & 0 & 0 & 0 \\ 1 & 0 & 0 & 0 \\ 0 & 0 & a_{2}^{\circ} & 1-a_{2}^{\circ} \\ 0 & 0 & a_{2}^{\circ} & 1-a_{2}^{\circ}\end{array}\right)$.

With our definition of $\mathbf{A}_{\wp}^{\circ}$, all its rows contain the onegeneration recursion for the reference value of their respective block, so that the elements of $\mathbf{A}_{\wp}^{\circ} \mathrm{Ep}^{\circ}(t)$ are the reference values in the next generation, that is, the elements of $\wp^{\circ}(t+1)$, and the right-hand side of Eq. (A.7) also vanishes. By construction, then, the unit eigenspace of $\mathbf{A}^{\circ}$ is entirely contained in the null space of $\mathbf{A}^{\circ}-\mathbf{A}_{\wp}^{\circ}$. Further, all eigenvalues of $\mathbf{A}^{\circ}-\mathbf{A}_{\wp}^{\circ}$ are $<1$ in modulus. This can be seen by writing this matrix as $(\mathbf{I}-\mathbf{C}) \mathbf{A}^{\circ}$ where $\mathbf{C}$ is a block diagonal matrix where the elements of each block are zero, except the column for the reference set of positions for the block, which elements are 1 . Then the eigenvalues of $\mathbf{I}-\mathbf{C}$ are found to be 0 or 1 . For any vector $\mathbf{x}$ represented as $\sum_{i} x_{i} \mathbf{b}_{i}$ in the basis of eigenvectors $\left(\mathbf{b}_{i}\right)$ of $\mathbf{A}^{\circ}$, with eigenvalues $0 \leq \lambda_{i} \leq 1$ (in modulus),

$$
\left(\mathbf{A}^{\circ}-\mathbf{A}_{\wp}^{\circ}\right) \mathbf{x}=\left(\mathbf{A}^{\circ}-\mathbf{A}_{\wp}^{\circ}\right) \sum_{i ; \lambda_{i}<1} x_{i} \mathbf{b}_{i}=(\mathbf{I}-\mathbf{C}) \sum_{i ; \lambda_{i}<1} \lambda_{i} x_{i} \mathbf{b}_{i},
$$

which confirms that $\mathbf{A}^{\circ}-\mathbf{A}_{\wp}^{\circ}$ is a strict contraction matrix.

\section{A.1.2. The result for any order of approximation}

Formally, the above construction does not depend on considering neutral expectations. That is, we redefine $\mathbf{v}(t) \equiv\left(\mathbf{v}_{Z}(t)\right)_{Z \in Z} \equiv$ $\left(E p_{Z}(t)-\wp_{Z}(t)\right)$ where the reference value for any set of positions $Z$ is the expected value (under selection) of the gamete frequency for gene copies at all loci in $Z$ being all taken in the same individual. We define $\mathbf{A}_{\wp}$ from $\mathbf{A}$ by the same row-replacement operation through which we defined $\mathbf{A}_{\wp}^{\circ}$. It remains to show that we still have $\mathbf{A} \boldsymbol{(}(t)=\boldsymbol{\wp}(t), \mathbf{A}_{\wp} \boldsymbol{\wp}(t)=\boldsymbol{\wp}(t)$, and there the argument needs to be modified since $\mathbf{A}$ no longer has the same block structure as $\mathbf{A}^{\circ}$. In the non-neutral matrix the recursion (hence the row of the matrix) for a focal set of positions, say $A B$, can involve effects from selection on other set(s) of positions, say on some locus $C$. Then the row for $A B$ involves non-zero elements for some columns standing for the non-focal set of loci $A B C$ (e.g., columns $A B C$ and $A B C / C$ may be nonzero), while in the neutral matrix recursions for $A B$ involve only sets of positions involving exactly loci $A$ and $B$. However, within each row (here the $A B$ row) the sum of coefficients for a given set of loci (here $A B C$ ) involving one or more additional loci is necessarily null (as noted in the main text, this can be seen by consideration of the case where the additional loci are not polymorphic). Thus if the reference vector has identical elements among all rows corresponding to each non-focal set of loci, these constant values for each non-focal set do not affect the elements of $\mathbf{A} \wp(t)$, nor those of $\mathbf{A}_{\wp} \wp(t)$, for the focal set.

From the above considerations, Eqs. (A.3) and (A.7) hold out of neutrality (o specifier removed), and

$\mathbf{d} \equiv \sum_{t=0}^{\infty} \mathbf{v}(t)=\sum_{t=0}^{\infty}\left(\mathbf{A}-\mathbf{A}_{\wp}\right)^{t} \mathbf{v}(0)=\mathbf{M}^{-1} \mathbf{v}(0)$

where

$\mathbf{M} \equiv \mathbf{I}-\mathbf{A}+\mathbf{A}_{\wp}$.

The eigenvalues of $\mathbf{A}-\mathbf{A}_{\wp}$ are all $<1$ (in modulus) in some neighborhood $\mathcal{N}$ of $\delta=0$ because those of $\mathbf{A}^{\circ}-\mathbf{A}_{\wp}^{\circ}$ are all $<1$ (in modulus). Thus all elements of the series $\sum_{t}\left(\mathbf{A}-\mathbf{A}_{\wp}\right)^{t}$ can be seen to converge uniformly in $\mathcal{N}$ and, assuming that the derivatives of $\left(\mathbf{A}-\mathbf{A}_{\wp}\right)$ up to given order $n$ are finite in $\mathcal{N}$, the $n$ th-order derivative of the fixation probabilities can be computed as the sum of derivatives of gamete frequency change over one generation.

Eq. (A.10) can hardly be used in its full exact form since it potentially involves very large matrices, needed for an exact description of the evolution of the population. However, it yields the $n$ th-order Taylor polynomial as

$\mathbf{d}^{[n]}=\left(\mathbf{M}^{-1}\right)^{[n]} \mathbf{v}(0)=\left(\sum_{k=0}^{n} \frac{\delta^{k}}{k !} \frac{d^{k} \mathbf{M}^{-1}}{d \delta^{k}}\right) \mathbf{v}(0)$,

which can be used in Eq. (34), and where reduced matrices can be substituted to $\mathbf{M}$ for computation of the low-order terms. For instance, the first derivative of $\mathbf{M}^{-1}$ with respect to $\delta$ is

$\frac{d \mathbf{M}^{-1}}{d \delta}=-\left(\mathbf{M}^{\circ}\right)^{-1} \frac{d \mathbf{M}}{d \delta}\left(\mathbf{M}^{\circ}\right)^{-1}$,

which yields the first-order Taylor polynomial

$\mathbf{d}=\left(\mathbf{I}-\mathbf{A}^{\circ}+\mathbf{A}_{\wp}^{\circ}\right)^{-1}\left[\mathbf{I}+\delta\left(\dot{\mathbf{A}}-\dot{\mathbf{A}}_{\wp}\right)\left(\mathbf{I}-\mathbf{A}^{\circ}+\mathbf{A}_{\wp}^{\circ}\right)^{-1}\right] \mathbf{v}(0)$,

where $\dot{\mathbf{A}}$ is the first-order perturbation of the row stochastic matrix $\mathbf{A}^{\circ}$, in which the row sums of perturbation terms are null, and $\dot{\mathbf{A}}_{\wp}$ is constructed from $\dot{\mathbf{A}}$ exactly in the same way as $\mathbf{A}_{\wp}$ is obtained from A; namely, $\dot{\mathbf{A}}_{\wp}=\mathbf{C} \dot{\mathbf{A}}$.

A.2. Cumulative moments of allelic states and mean first passage and sojourn times

\section{A.2.1. Fundamental matrix of a neutral system}

Our goal in this section is to express the elements of $\mathbf{d}^{\circ}$ and $\left(\mathbf{M}^{\circ}\right)^{-1}$ in terms of mean first passage and sojourn times. For ease of presentation of the argument, we will only consider a single diagonal block of the matrix $\mathbf{A}^{\circ}$ in the proofs below, as all the 
expressions hold with all matrices being diagonal block matrices where each block satisfy the given proofs (think of $\mathbf{A}^{\circ}$ as consisting of a single neutral system).

In our calculations we will use the so-called fundamental matrix $\mathbf{Z}$ associated to the transition matrix $\mathbf{A}^{\circ}$. For an absorbing Markov chain, the fundamental matrix is usually defined as (I $\mathbf{Q}^{-1}$, where $\mathbf{Q}$ is the transition matrix stripped of the rows and columns representing the absorbing states (Grinstead and Snell, 1997, p. 417). For a regular transition matrix, it is defined as

$\mathbf{Z} \equiv\left(\mathbf{I}-\mathbf{A}^{\circ}+\mathbf{A}_{\mathrm{c}}^{\circ}\right)^{-1}$

(Grinstead and Snell, 1997, p. 456), where $\mathbf{A}_{\mathrm{c}}^{\circ} \equiv \lim _{t \rightarrow \infty}\left(\mathbf{A}^{\circ}\right)^{t}$ is a matrix of which each row is a left eigenvector denoted $\mathbf{u}$ of $\mathbf{A}^{\circ}$ associated to its unit eigenvalue (i.e., $\mathbf{u}=\mathbf{u} \mathbf{A}^{\circ}$ ). For $\mathrm{a}$ regular Markov chain (one neutral system) $\mathbf{u}$ is uniquely defined as a probability vector, and when $\mathbf{A}^{\circ}$ consists of several neutral systems (several blocks) it is build up in a straightforward generalization of that case, being built from the probability (eigen)vectors for each of the regular Markov chains describing the recursions for a set of loci (block). A single neutral system may actually consist of an indecomposable Markov matrix (Iosifescu, 2007, p. 96), i.e., a matrix which includes both transient states and a single recurrent class, the latter further not always reduced to a singleton. For instance, the dynamics of the vector Ep $\equiv$ $\left(E p_{\mathrm{AB}}^{\circ}, \mathrm{E} p_{\mathrm{A} / \mathrm{B}}^{\circ}, \mathrm{E} p_{\mathrm{A} / \mathrm{AB}}^{\circ}, \mathrm{E} p_{\mathrm{A} / \mathrm{B} / \mathrm{A}}^{\circ}\right)$ is described by an indecomposable Markov matrix (see the recursions for this chain given by Eq. (28) and Eqs. (65)-(67)). To such matrices we will apply the definition of the fundamental matrix for regular chains, which is applicable to all mixing Markov matrices (Iosifescu, 2007, p. 133), thus including absorbing chains. This will serve us because the fundamental matrix can be expressed in terms of mean first passage and sojourn times, which will then be used to express $\left(\mathbf{M}^{\circ}\right)^{-1}$ in terms of such times by employing

$\left(\mathbf{M}^{\circ}\right)^{-1}=\mathbf{Z}-\mathbf{C Z}+\mathbf{C}$,

where (for each block) $\mathbf{C}$ is a matrix which elements are zero except the column for the reference set of positions for the block, which elements are 1 (see Eqs. (A.30)-(A.33)). We develop the argument for regular matrices first.

\section{A.2.2. Regular neutral systems}

A standard result for regular chains is that the mean first passage time $T_{S V}$, which is the expected number of steps it takes (looking backwards in time) for the set of gene lineages under focus, presently in the set of positions $S$, to reach the set of positions $V$, is given by

$T_{S V}=\frac{z_{V V}-z_{S V}}{u_{V}}$,

(Grinstead and Snell, 1997, p. 456-459). With this, the SVth element of the fundamental matrix of a regular chain can be expressed as

$z_{S V}=z_{V V}-T_{S V} u_{V}$

so that the $S V$ th element of $\mathbf{Z}-\mathbf{C Z}$ is

$z_{S V}-z_{R V}=\left(T_{R V}-T_{S V}\right) u_{V}$

which, along with $\mathbf{C v}(0)=\mathbf{0}$, allows us to write the Sth element of $\mathbf{d}^{\circ}=(\mathbf{Z}-\mathbf{C Z}+\mathbf{C}) \mathbf{v}^{\circ}(0)$ as

$d_{S}^{\circ}=\sum_{V \in \mathcal{V}}\left(T_{R V}-T_{S V}\right) u_{V}\left(p_{V}(0)-p_{R}(0)\right)$.

Because $\sum_{V \in \mathcal{V}} T_{S V} u_{V}$ is equal to a constant for all $S$ (Grinstead and Snell, 1997, p. 468, Levene and Loizou, 2002, p. 744), we finally have

$d_{S}^{\circ}=\sum_{V \in \mathcal{V}}\left(T_{R V}-T_{S V}\right) u_{V} p_{V}(0)$.

\section{A.2.3. Indecomposable neutral systems}

For indecomposable matrices, $\mathbf{A}^{\circ}$ can be represented in the standard way, as

$\mathbf{A}^{\circ}=\left(\begin{array}{ll}\mathbf{R} & \mathbf{0} \\ \mathbf{S} & \mathbf{Q}\end{array}\right)$,

where $\mathbf{R}$ describes an ergodic chain (here assumed regular) among recurrent states, $\mathbf{Q}$ collects the transition probabilities between the transient states, and $\mathbf{S}$ collects the transition probabilities from transient-to-recurrent states. For such an indecomposable matrix

$\lim _{t \rightarrow \infty}\left(\mathbf{A}^{\circ}\right)^{t}=\left(\begin{array}{ll}\mathbf{R}_{\mathrm{c}}^{\circ} & \mathbf{0} \\ \mathbf{R}_{\mathrm{c}}^{\circ} & \mathbf{0}\end{array}\right)$,

where $\mathbf{R}_{\mathrm{c}}^{\circ}$ is defined from $\mathbf{R}$ as previously described for regular matrices (Iosifescu, 2007, p. 126). Applying the definition of the fundamental matrix for regular matrices (Eq. (A.15)) to indecomposable matrices gives

$$
\begin{aligned}
\mathbf{Z} & \equiv\left(\mathbf{I}-\mathbf{A}^{\circ}+\mathbf{A}_{\mathrm{c}}^{\circ}\right)^{-1} \\
& =\left(\begin{array}{cc}
\left(\mathbf{I}-\mathbf{R}^{\circ}+\mathbf{R}_{\mathrm{c}}^{\circ}\right)^{-1} & \mathbf{0} \\
\left(\mathbf{I}-\mathbf{Q}^{-1} \mathbf{S}\left(\mathbf{I}-\mathbf{R}^{\circ}+\mathbf{R}_{\mathrm{c}}^{\circ}\right)^{-1}\right. & (\mathbf{I}-\mathbf{Q})^{-1}
\end{array}\right) .
\end{aligned}
$$

The diagonal blocks in $\mathbf{Z}$ are the fundamental matrices for the recurrent and transient states. The $S V$ th element of the recurrent block of $\mathbf{Z}$ is thus given by Eq. (A.18), while the $S V$ th element of the recurrent block of $\mathbf{Z}-\mathbf{C Z}$ is given by Eq. (A.19). The $S X$ th element of $(\mathbf{I}-\mathbf{Q})^{-1}$ is the mean time spent in state $X$ starting from state $S$ before absorption in the recurrent set (mean sojourn time). The $S V$ th element of the transient block of $\mathbf{Z}-\mathbf{C Z}$ is then equal to zero. Because the elements of $\mathbf{S}$ are the transition probabilities from transient states to recurrent states, the $S W$ th element of $(\mathbf{I}-\mathbf{Q})^{-1} \mathbf{S}$ is seen to contain the probabilities (denoted $\phi_{S V}$ ) that the chain starting from a transient state $S$ first reaches the recurrent set in state $W$ (i.e., the absorption probabilities). This leads to the $S V$ th element of the transient-to-recurrent block of $\mathbf{Z}$ being given by

$$
\begin{aligned}
\sum_{\operatorname{rec} W \in \mathcal{V}} \phi_{S W} z_{W V} & =\sum_{\operatorname{rec} W \in \mathcal{V}} \phi_{S W}\left(z_{V V}-T_{W V} u_{V}\right) \\
& =z_{V V}-\sum_{\operatorname{rec} W \in \mathcal{V}} \phi_{S W} T_{W V} u_{V},
\end{aligned}
$$

where the sum is over all recurrent states. The SV th element of the transient-to-recurrent block of $\mathbf{Z}-\mathbf{C Z}$ then becomes

$$
\sum_{\operatorname{rec} W \in \mathcal{V}} \phi_{S W} z_{W V}-\left(z_{V V}-T_{R V} u_{V}\right)=\left(T_{R V}-\sum_{\operatorname{rec} W \in \mathcal{V}} \phi_{S W} T_{W V}\right) u_{V} \text {. }
$$

Collecting terms, we have that for a recurrent state $S, d_{S}^{\circ}$ is given by Eq. (A.21), as in the regular case, while for a transient state $S, d_{S}^{\circ}$ is given by

$$
\begin{aligned}
d_{S}^{\circ}= & \sum_{\operatorname{rec} V \in \mathcal{V}}\left(T_{R V}-\sum_{\operatorname{rec} W \in \mathcal{V}} \phi_{S W} T_{W V}\right) u_{V} p_{V}(0) \\
& +\sum_{\text {trans } V \in \mathcal{V}} T_{S V}\left(p_{V}(0)-p_{R}(0)\right),
\end{aligned}
$$

where the second sum is over all transient states, in which $T_{S V}$ is defined as the mean time spent in $V$ starting from $S$ before entering the recurrent set (mean sojourn time). When the recurrent set consists of a single absorbing state (as occurs in a one-locus model or in a multilocus context without recombination), and that this absorbing state is taken to be the reference set, Eq. (A.27) simplifies to

$d_{S}^{\circ}=\sum_{\operatorname{trans} V \in \mathcal{V}} T_{S V}\left(p_{V}(0)-p_{R}(0)\right)$, 
where we used $\phi_{S R}=1$. Further, if there is initially a single mutant allele in the population for a one-locus model, $p_{V}(0)=0$ and $p_{R}(0)=1 / N$, whereby

$d_{S}^{\circ}=-\frac{1}{N} T_{S R}$,

where $T_{S R}=\sum_{\text {trans } V \in \mathcal{V}} T_{S V}$ is the mean time to absorption in $R$, given that a set of gene lineages initially resides in $S$ (Grinstead and Snell, 1997, p. 419); that is, the mean coalescence time.

\section{A.2.4. Expressing $\left(\mathbf{M}^{\circ}\right)^{-1}$ in terms of $\mathbf{Z}$}

In this Appendix, we express $\left(\mathbf{M}^{\circ}\right)^{-1}=\left(\mathbf{I}-\mathbf{A}^{\circ}+\mathbf{A}_{\wp}^{\circ}\right)^{-1}$ in terms of the fundamental matrix $\mathbf{Z}$ as defined in Eq. (A.15), which is applicable to all mixing Markov matrices (Iosifescu, 2007, p. 133). From the definition of $\mathbf{M}^{\circ}$, we have $\mathbf{I}-\mathbf{A}^{\circ}=\mathbf{M}^{\circ}-\mathbf{A}_{\wp}^{\circ}$ and on substitution into Eq. (A.15) gives $\mathbf{Z}=\left(\mathbf{M}^{\circ}+\mathbf{A}_{\mathrm{c}}^{\circ}-\mathbf{A}_{\wp}^{\circ}\right)^{-1}$. Leftmultiplying both sides of this equation by $\mathbf{M}^{\circ}+\mathbf{A}_{\mathrm{c}}^{\circ}-\mathbf{A}_{\wp}^{\circ}$, we have $\mathbf{M}^{\circ} \mathbf{Z}=\mathbf{I}-\left(\mathbf{A}_{\mathrm{c}}^{\circ}-\mathbf{A}_{\odot}^{\circ}\right) \mathbf{Z}$, and then right-multiplying by $\mathbf{Z}^{-1}$ and taking inverses, we obtain

$\left(\mathbf{M}^{\circ}\right)^{-1}=\mathbf{Z}\left(\mathbf{I}-\left(\mathbf{A}_{\mathrm{c}}^{\circ}-\mathbf{A}_{\wp}^{\circ}\right) \mathbf{Z}\right)^{-1}$.

For each block, the rows of the matrix $\left(\mathbf{A}_{\mathrm{c}}^{\circ}-\mathbf{A}_{\wp}^{\circ}\right) \mathbf{Z}$ are all identical and we can thus express this matrix in terms of the tensor product of two vectors. We can then apply the Sherman-Morrison formula (Bartlett, 1951, eq. 2), by which we obtain

$$
\left(\mathbf{I}-\left(\mathbf{A}_{\mathrm{c}}^{\circ}-\mathbf{A}_{\wp}^{\circ}\right) \mathbf{Z}\right)^{-1}=\mathbf{I}+\frac{\left(\mathbf{A}_{\mathrm{c}}^{\circ}-\mathbf{A}_{\wp}^{\circ}\right) \mathbf{Z}}{1+\tau},
$$

where $\tau$ is equal to the row sum of $\left(\mathbf{A}_{\mathrm{c}}^{\circ}-\mathbf{A}_{\circ}^{\circ}\right) \mathbf{Z}$. Because the row sums of $\mathbf{Z}$ are equal to one (Grinstead and Snell, 1997, p. 458; Iosifescu, 2007, p. 132), we have $\mathbf{Z A}_{\wp}^{\circ}=\mathbf{A}_{\wp}^{\circ}$ and $\mathbf{Z A}_{\mathrm{c}}^{\circ}=\mathbf{A}_{\mathrm{c}}^{\circ}$, so that insertion of Eq. (A.31) into Eq. (A.30) gives

$\left(\mathbf{M}^{\circ}\right)^{-1}=\mathbf{Z}+\frac{\left(\mathbf{A}_{\mathrm{c}}^{\circ}-\mathbf{A}_{\wp}^{\circ}\right) \mathbf{Z}}{1+\tau}$.

It now remains to evaluate $\tau, \mathbf{A}_{\mathrm{c}}^{\circ} \mathbf{Z}$ and $\mathbf{A}_{\wp}^{\circ} \mathbf{Z}$. From the definitions of $\mathbf{A}_{\mathrm{c}}^{\circ}$ and $\mathbf{Z}, \mathbf{A}_{\mathrm{c}}^{\circ} \mathbf{Z}=\mathbf{A}_{\mathrm{c}}^{\circ}$ and $\mathbf{I}-\mathbf{A}_{\mathrm{c}}^{\circ}=\left(\mathbf{I}-\mathbf{A}^{\circ}\right) \mathbf{Z}$ (Grinstead and Snell, 1997, p. 458-459). We have previously noted that $\mathbf{A}_{\wp}^{\circ}=\mathbf{C A}$, where (for each block) $C$ is a matrix which elements are zero except the column for the reference set of positions for the block, which elements are 1. Premultiplying $\mathbf{I}-\mathbf{A}_{\mathrm{c}}^{\circ}=\left(\mathbf{I}-\mathbf{A}^{\circ}\right) \mathbf{Z}$ by $\mathbf{C}$, one obtains

$\mathbf{A}_{\wp}^{\circ} \mathbf{Z}=\mathbf{C Z}-\mathbf{C}+\mathbf{A}_{\mathrm{c}}^{\circ}$,

which shows, first, that the row sums of $\mathbf{A}_{\wp}^{\circ} \mathbf{Z}$ are equal to one, as those of $\mathbf{A}_{\mathrm{c}}^{\circ}$, so that $\tau=0$ in Eq. (A.32); second, that $\left(\mathbf{M}^{\circ}\right)^{-1}=$ $\mathbf{Z}-\mathbf{C Z}+\mathbf{C}$.

\section{A.3. Explicit expression for Hill-Robertson effect $\Delta \pi_{\mathrm{HR}}$}

For third-order effects of selection we find that the reduction in the fixation probability of a mutant allele A due to interference is given by

$\Delta \pi_{\mathrm{HR}}=-s_{\mathrm{A}} s_{\mathrm{B}}^{2} p_{\mathrm{B}}(0)\left(1-p_{\mathrm{B}}(0)\right) \frac{X_{1}}{X_{2}}$,

where

$$
\begin{aligned}
X_{1}= & N^{2}\left[N(3 N-2)+(N-2)(N-1)(N+2) r-2(N-2)(N-1) r^{2}\right] \\
& \times\left[20-66 N+81 N^{2}-36 N^{3}-(N-1)(N(3 N(4 N-21)\right. \\
& \left.+88)-40) r+2(N-2)(N-1)^{2}(3 N-5) r^{2}\right]
\end{aligned}
$$

and

$$
\begin{aligned}
X_{2}= & 6 N((N-1) r+1)(3 N+(N-2)(N-1) r-2) \\
& \times\left[2(r-2) r^{2} N^{5}+r((44-17 r) r-26) N^{4}\right. \\
& +(r-1)(13 r(4 r-7)+18) N^{3} \\
& -(r-1)(r(73 r-134)+45) N^{2} \\
& \left.+4(r-1)(3 r-2)(4 r-5) N-12(r-1)^{3}\right] .
\end{aligned}
$$

When $r=0$, Eq. (A.34) reduces to Eq. (78) of the main text.

A.4. Two-locus model with social effects: Coevolution of helping and punishment

\section{A.4.1. Biological scenario}

In this Appendix, we present the first-order perturbations of fixation probabilities for a two-locus model where the alleles segregating in the population might have phenotypic effects on the fecundity of actors and receptors; namely, we consider social traits that result in frequency-dependent selection. We assume a helping and a punishment locus and that each individual interacts with its $N-1$ neighbors. We model interactions according to the strong-reciprocity protocol (e.g., Bowles and Gintis, 2004) in exactly the same way as detailed in Lehmann et al. (2007, eq. 5) for the interactions taking place among individuals within patches of size $N$. Bearing a mutant helping allele at locus $A$ causes the actor to provide a benefit $B_{\mathrm{H}} /(N-1)$ to a single partner at a direct fecundity $\operatorname{cost} C_{\mathrm{H}} /(N-1)$ to himself. Individuals bearing a mutant punishment allele at the $B$ locus express an act of punishment at a cost $C_{\mathrm{P}} /(N-1)$ to self, conditional on their partners not having expressed helping, which decreases the fitness of the recipient by $D_{\mathrm{P}} /(N-1)$. Individuals bearing the resident alleles at each locus take the benefits and pay no direct costs. With these assumptions, the relative fecundity of individual $i$ is given by

$$
\begin{aligned}
f_{i}= & +\frac{1}{N-1} \sum_{k, k \neq i}\left[-C_{\mathrm{H}} p_{\mathrm{A}(i)}+B_{\mathrm{H}} p_{\mathrm{A}(k)}-C_{\mathrm{P}}\left(1-p_{\mathrm{A}(k)}\right) p_{\mathrm{B}(i)}\right. \\
& \left.-D_{\mathrm{P}}\left(1-p_{\mathrm{A}(i)}\right) p_{\mathrm{B}(k)}\right] .
\end{aligned}
$$

With this fecundity function we can compute the fitness of individual $i$ as $w_{i}=f_{i} / f$, where the average fecundity is given by $f=1+\left(B_{\mathrm{H}}-C_{\mathrm{H}}\right) p_{\mathrm{A}}-\left(C_{\mathrm{P}}+D_{\mathrm{P}}\right)\left(p_{\mathrm{B}}-p_{\mathrm{A} / \mathrm{B}}\right)$. Inserting the fitness function into Eq. (43) and assuming that $C_{\mathrm{H}}, B_{\mathrm{H}}, C_{\mathrm{P}}$, and $D_{\mathrm{P}}$ are of order $\delta$, the recursion for the conditional frequency of the mutant allele a locus $U(U=A$ or $U=B)$ can be written to the first-order in selection strength as

$$
\begin{aligned}
\mathrm{E} \cdot\left[p_{U}^{\prime} \mid \mathbf{p}\right]= & p_{U}-C_{\mathrm{H}} p_{\mathrm{A} U}+B_{\mathrm{H}} p_{\mathrm{A} / U}-C_{\mathrm{P}}\left(p_{\mathrm{B} U}-p_{\mathrm{B} U / \mathrm{A}}\right) \\
& -D_{\mathrm{P}}\left(p_{\mathrm{B} / U}-p_{\mathrm{A} / \mathrm{B}}\right)-\left(B_{\mathrm{H}}-C_{\mathrm{H}}\right) p_{\mathrm{A}} p_{U} \\
& -\left(C_{\mathrm{P}}+D_{\mathrm{P}}\right)\left(p_{\mathrm{B}} p_{U}-p_{\mathrm{A} / \mathrm{B}} p_{U}\right) .
\end{aligned}
$$

Integration over all sample paths then allows us to obtain the recursion for the unconditional frequency of the helping and punishment alleles, respectively, as

$$
\begin{aligned}
& \mathrm{E} p_{\mathrm{A}}{ }^{\prime}=\mathrm{E} p_{\mathrm{A}}^{\cdot}-C_{\mathrm{H}} \mathrm{E} p_{\mathrm{A}}^{\circ}+B_{\mathrm{H}} \mathrm{E} p_{\mathrm{A} / \mathrm{A}}^{\circ}-C_{\mathrm{P}}\left(\mathrm{E}_{\mathrm{AB}}^{\circ}-\mathrm{E} p_{\mathrm{A} / \mathrm{AB}}^{\circ}\right) \\
& -\left(B_{\mathrm{H}}-C_{\mathrm{H}}\right) \mathrm{E}_{\mathrm{A} / \mathrm{A}}^{\circ}+\left(C_{\mathrm{P}}+D_{\mathrm{P}}\right)\left(\mathrm{E}_{\mathrm{A} / \mathrm{B}}^{\circ}-\mathrm{E} p_{\mathrm{A} /(\mathrm{A} / \mathrm{B})}^{\circ}\right)
\end{aligned}
$$

and

$$
\begin{aligned}
\mathrm{Ep}_{\mathrm{B}}^{{ }^{\prime}=} & \mathrm{E} p_{\mathrm{B}}^{\circ}-C_{\mathrm{H}} \mathrm{E} p_{\mathrm{AB}}^{\circ}+B_{\mathrm{H}} \mathrm{E} p_{\mathrm{A} / \mathrm{B}}^{\circ}-C_{\mathrm{P}}\left(\mathrm{E} p_{\mathrm{B}}^{\circ}-\mathrm{E} p_{\mathrm{A} / \mathrm{B}}^{\circ}\right) \\
& -D_{\mathrm{P}}\left(\mathrm{E}_{\mathrm{B} / \mathrm{B}}^{\circ}-\mathrm{E} p_{\mathrm{AB} / \mathrm{B}}^{\circ}\right)-\left(B_{\mathrm{H}}-C_{\mathrm{H}}\right) E p_{\mathrm{A} / \mathrm{B}}^{\circ} \\
& +\left(C_{\mathrm{P}}+D_{\mathrm{P}}\right)\left(\mathrm{E}_{\mathrm{B} / \mathrm{B}}^{\circ}-\mathrm{E}^{\circ}{ }_{\mathrm{B} /(\mathrm{A} / \mathrm{B})}^{\circ}\right),
\end{aligned}
$$


where all neutral moments of allelic states have already been encountered in the main text (see Eqs. (53)-(54) and Eqs. (65)(67)). These equations then allow us to construct $\mathbf{A}$ for both the mutant helping and punishment alleles.

\section{A.4.2. Results}

We find that the first-order perturbation of the fixation probability of a single mutant helping allele $\left(p_{\mathrm{A}}(0)=1 / N\right)$ is a complicated function of the model parameters and in particular of the recombination rate $r$ and the initial frequency $p_{B}(0)$ of the punishment allele. When a single helping allele arises on a single mutant punishment background $\left(p_{\mathrm{B}}(0)=1 / N\right)$, the first-order perturbation simplifies to

$$
\begin{aligned}
\dot{\pi}_{\mathrm{A}}= & -C_{\mathrm{H}}-\frac{B_{\mathrm{H}}-C_{\mathrm{H}}}{N} \\
& +\frac{\left(D_{\mathrm{P}}-C_{\mathrm{P}}\right)\left(N+r(N-1)^{2}\right)-C_{\mathrm{P}}(N-2)}{\{1+r(N-1)\}\{3 N-2+r(N-2)(N-1)\}},
\end{aligned}
$$

which is a decreasing function of the recombination rate. The second term on the right-hand side is a cost, while the third term is a benefit, which is decreasing when population size increases, and is likely to dominate the cost. For instance, in the absence of recombination $(r=0)$ and when population size becomes very large (tends to infinity), the cost vanishes while the benefit reduces to $2\left(D_{\mathrm{P}}-2 C_{\mathrm{P}}\right) / 3$.

We find that the average perturbation of the fixation probability of a single mutant (over the two backgrounds at locus B on which the mutant may appear) is given by

$\dot{\bar{\pi}}_{\mathrm{A}}=-C_{\mathrm{H}}-\frac{B_{\mathrm{H}}-C_{\mathrm{H}}}{N}+p_{\mathrm{B}}(0)\left(D_{\mathrm{P}}-\frac{D_{\mathrm{P}}+C_{\mathrm{P}}}{N}\right)$,

which is increasing in population size but independent of the recombination rate. Hence, finite population size decreases the selective pressure on helping once it is taken into account that the helping allele may appear on two different backgrounds. Letting population size become very large, the right-hand side of Eq. (A.42) then reduces to the gradient of selection on gene frequency change found in deterministic models with basic structure similar to the present stochastic one (Lehmann et al., 2007, eq. 17, see also Gardner et al., 2007).

The perturbation of the fixation probability of a single mutant punishment allele at locus $B$ when it arises on a single mutant background at locus $A$ is given by

$$
\begin{aligned}
\dot{\pi}_{\mathrm{B}} & =-C_{\mathrm{P}}+\frac{D_{\mathrm{P}}+C_{\mathrm{P}}}{N}+\left(-C_{\mathrm{H}}-\frac{B_{\mathrm{H}}-C_{\mathrm{H}}}{N}\right) /(1+r(N-1)) \\
& +\left(\frac{N-1}{N}\right) \frac{\left(D_{\mathrm{P}}+C_{\mathrm{P}}\right)(N-2-2 r(N-1))+C_{\mathrm{P}} r N^{2}}{\{1+r(N-1)\}\{3 N-2+r(N-2)(N-1)\}},
\end{aligned}
$$

and the average perturbation of the fixation probability of a single punishment allele (over the two backgrounds at locus A on which the mutant may appear) is

$\dot{\bar{\pi}}_{\mathrm{B}}=\left(1-p_{\mathrm{A}}(0)\right)\left(-C_{\mathrm{P}}+\frac{D_{\mathrm{P}}+C_{\mathrm{P}}}{N}\right)$,

which is a decreasing function of population size and again independent of the recombination rate. As was observed for helping, letting population size become large, the right-hand side of Eq. (A.44) then reduces to the gradient of selection on gene frequency change in deterministic models for the evolution of punishment (Lehmann et al., 2007, eq. 20, see also Gardner et al., 2007).

In Fig. 3 we checked these analytical results with those obtained from simulations by comparing the predicted perturbations given by Eqs. (A.41) and (A.43) with observed ones. This figure illustrates that the change of the direction of selection as a function of the recombination rate is well predicted by the firstorder perturbations even when selection is not weak, although discrepancies arise when selection is very strong.

\section{References}

Bartlett, M.S., 1951. An inverse matrix adjustment arising in discriminant analysis Annals of Mathematical Statistics 22, 107-111.

Bartlett, M.S., 1955. An Introduction to Stochastic Processes. Cambridge University Press, Cambridge.

Barton, N.H., 1995. Linkage and the limits to natural selection. Genetics 140, 821-841.

Barton, N.H., Otto, S.P., 2005. Evolution of recombination due to random drift Genetics 169, 2353-2370.

Bowles, S., Gintis, H., 2004. The evolution of strong reciprocity: Cooperation in heterogeneous populations. Theoretical Population Biology 65, 17-28.

Christiansen, F.B., 1999. Population Genetics of Multiple Loci. John Wiley, New York

Crow, J.F., Kimura, M., 1970. An Introduction to Population Genetics Theory. Harper and Row, New York.

Ewens, W.J., 2004. Mathematical Population Genetics. Springer-Verlag, New York.

Gardner, A., West, S.A., Barton, N., 2007. The relation between multilocus population genetics and social evolution theory. American Naturalist 169, 207-226.

Geritz, S.A.H., Kisdi, E., Meszéna, G., Metz, J.A.J., 1998. Evolutionarily singular strategies and the adaptive growth and branching of the evolutionary tree. Evolutionary Ecology 12, 35-57.

Gillespie, J.H., 2004. Population Genetics: A Concise Guide. Johns Hopkins, Baltimore, London.

Grafen, A., 2007. An inclusive fitness analysis of a network model of Ohtsuki etal. Journal of Evolutionary Biology 20, 2278-2283.

Grinstead, C.M., Snell, J.L., 1997. Introduction to Probability, 2nd ed. American Mathematical Society, Providence, RI, Available online at http://www.dartmouth. edu/ chance/teaching_aids/books_articles/probability_book/book.html.

Guillaume, F., Perrin, N., 2006. Joint evolution of dispersal and inbreeding load Genetics 173, 497-509.

Haldane, J.B.S., 1927. A mathematical theory of natural and artificial selection V. Selection and mutation.. Proceedings of the Cambridge Philosophical Society 23, 838-844.

Hamilton, W.D., 1964. The genetical evolution of social behaviour, I. Journal of Theoretical Biology 7, 1-16.

Hamilton, W.D., 1970. Selfish and spiteful behavior in an evolutionary model Nature 228, 1218-1220.

Hamilton, W.D., May, R.M., 1977. Dispersal in stable habitats. Nature 269, 578-581.

Hartl, D., Clark, A.G., 2007. Principles of Population Genetics, 4th ed. Sinauer, Massachusetts.

Hill, W., 1972. Probability of fixation of genes in populations of variable size Theoretical Population Biology 3, 27-40.

Hill, W., Robertson, A., 1966. The effects of linkage and the limits to artificial selection. Genetical Research 8, 269-294.

Iosifescu, M., 2007. Finite Markov Processes and Their Applications. Dover, New York.

Karlin, S., 1968. Equilibrium behavior of population genetic models with nonrandom mating: Part II: Pedigrees, homozygosity and stochastic models. Journal of Applied Probability 5, 487-566.

Karlin, S., McGregor, J., 1968. Rates and probabilities of fixation for two locus random mating finite populations without selection. Genetics 58, 141-159.

Kimura, M., 1963. A probability method for treating inbreeding systems, especially with linked genes. Biometrics 19, 1-17.

Kirkpatrick, M., Johnson, T., Barton, N., 2002. General models of multilocus evolution. Genetics 161, 1727-1750.

Kurokawa, S., Ihara, Y., 2009. Emergence of cooperation in public goods games. Proceedings of the Royal Society of London Series B-Biological Sciences 276, 1379-1384.

Le Galliard, J., Ferrière, R., Dieckmann, U., 2003. The adaptive dynamics of altruism in spatially heterogeneous populations. Evolution 57, 1-17.

Lehmann, L., 2008. The adaptive dynamics of niche constructing traits in spatially subdivided populations: Evolving posthumous extended phenotypes. Evolution $62,549-566$.

Lehmann, L., Rousset, F., Roze, D., Keller, L., 2007. Strong reciprocity or strong ferocity? A population genetic view of the evolution of altruistic punishment The American Naturalist 170, 21-36.

Lessard, S., Ladret, V., 2007a. Fixation probability for a beneficial allele and a mutant strategy in a linear game under weak selection in a finite island model. Theoretical Population Biology 72, 409-425.

Lessard, S., Ladret, V., 2007b. The probability of fixation of a single mutant in an exchangeable selection model. Journal of Mathematical Biology 54 721-744.

Leturque, H., Rousset, F., 2002. Dispersal, kin competition, and the ideal free distribution in a spatially heterogeneous population. Theoretical Population Biology 62, 169-180.

Levene, M., Loizou, G., 2002. Kemeny's constant and the random surfer. American Mathematical Monthly 109, 741-745.

Ohta, T., 1968. Effect of linkage disequilibrium and epistasis on fixation probability in small populations with two segregating loci.. Theoretical and Applied Genetics 38, 243-248.

Ohtsuki, H., Nowak, M.A., 2006. Evolutionary games on cycles. Proceedings of the Royal Society of London Series B-Biological Sciences 273, 2249-2256. 
Otto, S., Barton, N., 2001. Selection for recombination in small populations Evolution 55, 1921-1931.

Otto, S.P., Barton, N.H., 1997. The evolution of recombination: Removing the limits to natural selection. Genetics $147,879-906$

Pen, I., 2000. Reproductive effort in viscous populations. Evolution 54, 293-297.

Rousset, F., 2003. A minimal derivation of convergence stability measures. Journal of Theoretical Biology 221, 665-668.

Rousset, F., 2004. Genetic Structure and Selection in Subdivided Populations. Princeton University Press, Princeton, NJ.

Rousset, F., Billiard, S., 2000. A theoretical basis for measures of kin selection in subdivided populations: Finite populations and localized dispersal. Journal of Evolutionary Biology 13, 814-825.

Roze, D., 2009. Diploidy, population structure and the evolution of recombination. American Naturalist (in press).

Roze, D., Rousset, F., 2008. Multilocus models in the infinite island model of population structure. Theoretical Population Biology 73, 529-542.
Singh, M., 1990. nth-order derivatives of certain inverses and the Bell polynomials. Journal of Physics A: Mathematical and General 23, 2307-2313.

Slatkin, M., 1971. On treating the chromosome as the unit of selection. Genetics 72 , 157-168.

Slatkin, M., 1991. Inbreeding coefficients and coalescence times times. Genetical Research 58, 167-175

Taylor, P.D., 1992. Altruism in viscous populations - An inclusive fitness model. Evolutionary Ecology 6, 352-356.

Wild, G., Taylor, P., 2004. Fitness and evolutionary stability in game theoretic models of finite populations. Proceedings of the Royal Society of London Series BBiological Sciences 271, 2345-2349.

Wolfram, S., 2003. Mathematica, 5th ed. Cambridge University Press, Cambridge.

Wright, S., 1931. Evolution in Mendelian populations. Genetics 16, 97-159.

Wright, S., 1969. Evolution and the Genetics of Populations II. The Theory of Gene Frequencies. University of Chicago Press, Chicago. 